\title{
Phase Transitions and Reflection Positivity. I. General Theory and Long Range Lattice Models
}

\author{
Jürg Fröhlich ${ }^{1 \star \star \star}$, Robert Israel ${ }^{2 \star \star \star}$, Elliot H. Lieb ${ }^{3 \dagger}$, and Barry Simon ${ }^{3 \star \star}$ \\ 1 Department of Mathematics, Princeton University, Princeton, NJ 08540, USA \\ 2 Department of Mathematics, University of British Columbia, Vancouver, B.C., Canada \\ 3 Departments of Mathematics and Physics, Princeton University, Princeton, NJ 08540, USA
}

\begin{abstract}
We systematize the study of reflection positivity in statistical mechanical models, and thereby two techniques in the theory of phase transitions: the method of infrared bounds and the chessboard method of estimating contour probabilities in Peierls arguments. We illustrate the ideas by applying them to models with long range interactions in one and two dimensions. Additional applications are discussed in a second paper.
\end{abstract}

\section{Introduction}

Among the recent developments in the rigorous theory of phase transitions have been the introduction of two powerful techniques motivated in part by ideas from constructive quantum field theory: the method of infrared bounds $[10,4]$ which provides the only presently available tool for proving that phase transitions occur in situations where a continuous symmetry is broken, and the chessboard estimate method of estimating contour probabilities in a Peierls' argument [14, 9]. This is the first of three papers systematizing, extending and applying these methods. In this paper, we present the general theory and illustrate it by considering phase transitions in one and two dimensional models with long range interactions. In II, [7], we will consider a large number of applications to lattice models and in III, [8] some continuous models including Euclidean quantum field theories. Reviews of some of our ideas and those in $[4,9,10,14]$ can be found in $[5,6,23,27,43]$. An application can be found in [19].

Three themes are particularly emphasized in these papers. The first, $\$ \S 2-4$, is the presentation of a somewhat abstract framework, partly for clarification (e.g.

\footnotetext{
* Present address: Institute des Hautes Études Scientifiques, 35, Route de Chartres, F-91440 Buressur-Yvette, France

$\star \star \quad$ Research partially supported by US National Science Foundation under Grant MPS-75-11864

$\star \star \star$ Research partially supported by Canadian National Research Council under Grant A4015

$+\quad$ Research partially supported by US National Science Foundation under Grant MCS-75-21684$\mathrm{A} 01$
} 
the tricks in [4] to handle the quantum antiferromagnet may appear more natural in the light of $\$ \$ 2,3$ below) but mainly for the extensions of the theory thereby suggested (e.g. the second theme below and the use, for classical systems, of reflections in planes containing sites: this idea, occurring already in [9], will be critical for many of our applications, e.g. to the classical antiferromagnets in external field). The abstract framework also clarifies various limitations of the theory such as its present inapplicability to the quantum Heisenberg ferromagnets and its restriction to reflections in planes between lattice planes for quantum systems. The second theme is the extension of the methods beyond the nearest neighbor simple cubic models emphasized in $[10,4,9]$. It will turn out $(\S 3)$ that rather few additional short range interactions can be accomodated but that a larger variety of long range interactions can be treated. This extension will allow us $(\S 5)$ to recover and extend to suitable quantum models the results of Dyson [3] (resp. Kunz-Pfister [26]) on long range one (resp. two) dimensional systems. It will also allow us (see II) to discuss a number of lattice Coulomb gases: for example, a "hard core model" where each site can have charge $0,+1$ or -1 will have two "crystal phases" for sufficiently low temperatures and large fugacity and, for sufficiently low temperatures and suitable fugacity, a third phase which can be thought of as a "plasma" or "gas" phase. Finally it will allow us to construct (see III) a $t w o$ dimensional quantum field theory (a $\phi^{4}$ perturbation of a generalized free field) with a spontaneously broken continuous symmetry.

For pair interactions, Hegerfeldt and Nappi [18] have proposed our sufficient condition for reflection positivity but they did not discuss the connection with phase transitions or the quantum case; see also their footnote on p. 4 of their paper.

The final theme involves the development of an idea in $[10,5]$ for proving that phase transitions occur in a situation where there is no symmetry broken and thus no a priori clear value of external field or fugacity for the multiple phase point. In all cases, the value can be computed for zero-temperature and one shows that there are multiple phases at some nearby value for low temperature, although our methods do not appear to specify the value by any computationally explicit procedure. This technique, which we do not discuss until Paper II, allows us in particular to recover some results of Pirogov-Sinai [33-35] including the occurrence of transitions in the triangle model (ordinary Ising ferromagnet in external field but with an additional interaction $K \sum \sigma_{i} \sigma_{j} \sigma_{k}$ over all triples $i j k$ where $i$ and $k$ are nearest neighbors of $j$ in orthogonal directions) and the occurrence of three phases in the Fisher stabilized antiferromagnet in suitable magnetic field (ordinary Ising antiferromagnet but with additional next nearest neighbor ferromagnetic coupling). As another example we mention an analysis of some models of Ginibre, discussed by Kim-Thompson [32] in the mean field approximation, with the property that at low temperatures there are an infinite number of external field values with multiple phases.

Next we want to make some remarks on the limitations, advantages and disadvantages of the reflection positivity (RP) methods. As regards the chessboard Peierls argument, it is useful to compare it with the most sophisticated Peierls type method that we know of, that of Pirogov-Sinai (PS method) [33-35, 20] (a comparison with the "naive" Peierls argument can be found in [27]): 
1) The most serious defect in the RP method is that the requirement of reflection positivity places rather strong restrictions on the interactions, especially for finite range interactions. For example, the PS analysis of the Fisher antiferromagnet would not be affected if one added an additional ferromagnetic coupling $\sigma_{i} \sigma_{j}$ for pairs $i j$ with $i-j=(8,10)$ (for example) while our argument would be destroyed no matter how small the coupling! More significantly, the RP analysis in this case requires that $\sigma_{(0,0)} \sigma_{(1,1)}$ and $\sigma_{(0,0)} \sigma_{(1,-1)}$ have equal couplings; PS does not. Similarly in the triangle model, an RP argument requires the four kinds of triangles to have equal couplings while PS does not.

2) RP can handle certain, admittedly special, long range couplings, among them interactions of physical interest such as Coulomb monopole and dipole couplings. PS in its present form is restricted to finite range interactions.

3) Inherent in the PS method is the notion that one is looking at a system with a "finitely degenerate ground state". This is not inherent in the RP method: all that is important is that a finite number of specific periodic states have a larger internal energy per unit volume than the true ground states. In some cases, e.g. the antiferromagnet without Fisher stabilization, there is no practical difference since the finite number of states of importance in RP are among the infinitely many ground states that prevent the application of PS. However, there is a model (of a liquid crystal) with an infinitely degenerate ground state to which Heilmann and Lieb [19] have applied the RP method with success. This model has only two ground states in finite volume with suitable boundary conditions, but infinitely many ground states in the PS sense in infinite volume.

4) The PS method gives much more detailed information than the RP method on the manifold of coexisting phases. For example in the Fisher antiferromagnet, there is, for $T$ small, an external field, $\mu(T)$, near the computable number $\mu(0)$, so that there are three (or more) phases at that value of $T$ and $\mu$. PS obtain continuity of $\mu(T)$ in $T$ while RP does not, but shows only that $\mu(T) \rightarrow \mu(0)$ as $T \rightarrow 0$.

5) While neither PS nor we have tried hard to optimize the lower bounds on transition temperatures, it seems reasonably clear that RP methods would produce better bounds.

6) PS require the number of values that a given spin takes to be finite. RP methods effortlessly extend to models like the anisotropic classical Heisenberg model (see [9]).

7) PS can only handle classical models, at least in its present version. RP methods can handle certain quantum models quite efficiently (see [9]).

8) RP works most naturally for states with periodic boundary conditions. This can occasionally be awkward.

9) PS obtain the exact number of phases at the maximum phase points while RP only yields a lower bound. This difference is probably not intrinsic, and RP methods could probably be combined with [11] to yield the exact number of phases.

10) To our, admittedly biased, tastes the RP method seems considerably simpler than the PS method.

As regards the infrared bounds method, there is no comparable method with which to compare it, but we note it is most unfortunate that the only available 
method for proving phase transitions depends so strongly on reflection positivity. We mention two examples to illustrate this remark:

1) In [10], it is proven that the classical Heisenberg ferromagnet with nearest neighbor interaction has a phase transition for a simple cubic lattice. The methods of $\$ \$ 2-4$ easily extend this result to face centered cubic and many other lattices, but not to the body centered cubic lattice. This remains an open problem.

2) There has been some discussion recently (see [36] and references therein) of an intriguing model, originally due to Elliott [28], which should have "helical" long range order: consider a one dimensional plane rotor or $N$-vector, $N \geqq 3$ model with nearest neighbor ferromagnet coupling, $J$, and somewhat stronger second neighbor antiferromagnet coupling, $K$. It will have a helical ground state, i.e. in a ground state $\sigma_{i} \cdot \sigma_{i+1}=\cos \theta$ for some $\theta \neq 0, \pi$ depending on the exact value of $J / K$. Of course, this helical ordering won't persist to finite temperature in the one dimensional case, but if one adds two more dimensions with conventional nearest neighbor ferromagnetic couplings one expects helical order will persist. We do not see how to prove this with RP methods; indeed, infrared bounds obtained by RP methods always seem to blow up at a single $p$ while at least two $p$ 's are involved here due to the evenness of the function $E_{p}$. We note that if one could prove an infrared bound, helical order would be proven since $E_{p}$ vanishes at precisely two $p$ 's with a zero of order $p^{2}$.

Finally, we summarize the contents of the remaining sections. In $\$ 2$, we present an abstract framework for reflection positivity and provide the basic perturbation criteria which allow one to go from reflection positivity for uncoupled spins to reflection positivity for suitably coupled spins. In $\$ 3$, we specialize to spin systems and examine two questions: about what kinds of planes does one have reflection positivity for the system of uncoupled spins, and what kinds of interactions obey the basic perturbation criteria of $\$ 2$ ? In $\$ 4$, we review and describe the two basic RP methods of proving phase transitions when one has reflection positivity about the large family of planes obtained by translating a basic family of planes. In $\$ 5$, we discuss the applications to recover the Dyson and Kunz-Pfister results already mentioned.

\section{Abstract Theory of Reflection Positivity}

Reflection positivity was introduced in quantum field theory by Osterwalder and Schrader [30] and it has continued to play an important role there. Its significance in the study of phase transitions for lattice gases was realized in $[10,5,9]$, although we must emphasize that transfer matrix ideas are intimately connected with reflection positivity. Klein [25] has considered other abstractions in somewhat different contexts.

To understand the framework we are about to describe, it is useful to keep in mind a particular example, describing a chain of Ising spins, that is essentially that given in $[10,9]$ (we describe the example after the basic framework).

$\mathfrak{Z}$ will be a real algebra (with unit) of observables. (We note that to say $\mathfrak{A}$ is a real algebra does not preclude $\mathfrak{Y}$ from being, say, an algebra of complex valued functions: "real" means that we only suppose that one can multiply by real scalars.) Below we will freely use and expand exponentials and use the Trotter- 
product formula (in cases where $\mathfrak{A}$ is non-abelian). In most applications these manipulations present no problem since $\mathfrak{P}$ is usually finite dimensional. In III, we will deal with some unbounded operators and exercise some care on this point. We suppose we are given a linear functional $A \rightarrow\langle A\rangle_{0}$ on $\mathfrak{A}$ with $\langle 1\rangle_{0}=1$. Given $H \in \mathfrak{U}$, we define

$$
\langle A\rangle_{H}=\left\langle A e^{-H}\right\rangle_{0} /\left\langle e^{-H}\right\rangle_{0} .
$$

Moreover, we suppose $\mathfrak{A}$ contains two subalgebras $\mathfrak{U}_{+}$and $\mathfrak{H}_{-}$and a real linear morphism $\theta: \mathfrak{H}_{+} \rightarrow \mathfrak{U}_{-}$. [The phrase "real linear" does not preclude $\theta$ from being complex linear or complex antilinear; morphism means $\theta(A B)=\theta(A) \theta(B)$. In most examples, $\theta$ has an extension to $\mathfrak{H}_{+} \cup \mathfrak{U}_{-}$obeying $\theta^{2}=1$, but this property plays no role in our considerations below.]

The example to keep in mind involves $2 n$ spin $1 / 2$-Ising spins $\sigma_{-n+1}$, $\sigma_{-n+2}, \ldots, \sigma_{n}$. Then $\mathfrak{Q}$ is the family of polynomials in all the $\sigma$ 's, $\mathfrak{U}_{+}\left(\right.$resp. $\left.\mathfrak{H}_{-}\right)$the polynomials in $\sigma_{1}, \ldots, \sigma_{n}$ (resp. $\left.\sigma_{0}, \sigma_{-1} \ldots \sigma_{-n+1}\right)$, and $\theta$ is defined so that $\theta\left(\sigma_{i}\right)$ $=\sigma_{-i+1} ;\langle A(\sigma)\rangle_{0}=\frac{1}{4^{n}} \sum_{\sigma_{l}= \pm 1} A\left(\sigma_{i}\right)$. Although $\mathfrak{U}_{+}$and $\mathfrak{U}_{-}$have trivial intersection in this example, we will not suppose this to be true in the abstract setting; we will not even suppose that $\mathfrak{U}_{+}$and $\mathfrak{U}_{-}$commute with each other, although it will turn out that there are no cases for which we can prove perturbed reflection positivity with non-mutually-commuting $\mathfrak{H}_{+}$and $\mathfrak{U}_{-}$(with the exception of some Fermion systems).

Definition. A real linear functional $\langle\cdot\rangle$ on $\mathfrak{U}$ is called reflection positive $(\mathrm{RP})$ if and only if $\langle A \theta(A)\rangle \geqq 0$ for all $A \in \mathfrak{H}_{+}$.

The reader should check RP and GRP (defined below) for the functional $\langle\cdot\rangle_{0}$ in the example. Unfortunately, we know of no abstract perturbation theory for functionals satisfying RP in the fully non-commutative setting, but a slightly stronger notion is preserved under suitable perturbations:

Definition. $\langle\cdot\rangle$ is called generalized reflection positive (GRP) if and only if

$$
\left\langle A_{1} \theta\left(A_{1}\right) \ldots A_{m} \theta\left(A_{m}\right)\right\rangle \geqq 0
$$

for all $A_{1}, \ldots, A_{m} \in \mathfrak{U}_{+}$.

Theorem 2.1. If $-H=B+\theta(B)+\sum_{j=1}^{k} C_{i} \theta\left(C_{i}\right)$ (or more generally $B+\theta(B)+\int C(x)$ $0[C(x)] d \varrho(x)$ for a positive measure dQ) with $B, C_{i} \in \mathfrak{U}_{+}$and if $\langle\cdot\rangle_{0}$ is $G R P$, then $\langle\cdot\rangle_{H}$, defined in (2.1) is GRP.

Proof. For simplicity, let us consider first the case where $\mathfrak{A}$ is abelian even though it is a special case of the general situation we then discuss. Then, since $\theta$ is a morphism

$$
e^{-H}=e^{B} \theta\left(e^{B}\right) e^{\Sigma C_{2} \theta\left(C_{2}\right)} .
$$

Expanding the exponential, we see that

$$
e^{-H}=\text { sum of terms of the form }\left(D_{1} \theta\left(D_{1}\right) \ldots D_{j} \theta\left(D_{j}\right)\right) \text {, }
$$

so that by GRP for $\langle\cdot\rangle_{0},\left\langle e^{-H}\right\rangle_{0} \geqq 0$ and $\left\langle e^{-H} A_{1} \theta\left(A_{1}\right) \ldots A_{m} \theta\left(A_{m}\right)\right\rangle_{0} \geqq 0$. 
For the general non-abelian case, we first use the Trotter product formula to write

$$
e^{-H}=\lim _{k \rightarrow \infty}\left[e^{B / k} \theta\left(e^{B / k}\right) \prod_{i} e^{C_{l} \theta\left(C_{1}\right) / k}\right]^{k}
$$

and then expand to get $e^{-H}$ as a limit of sums of $\pi\left[D_{j} \theta\left(D_{j}\right)\right]$.

In the next section, we will give a relevant example (Example 6) of a situation with $\langle\cdot\rangle_{0}$ RP but not GRP. There is one case where RP implies GRP (this, in fact, is the only case for which we know how to prove GRP!):

Theorem 2.2. If $\mathfrak{U}_{+}$and $\mathfrak{H}_{-}$commute with each other, a linear functional is $R P$ if and only if it is GRP.

Proof. $\pi A_{i} \theta\left(A_{i}\right)=\left(\pi A_{i}\right) \theta\left(\pi A_{i}\right)$ since the $A_{j}$ and $\theta\left(A_{i}\right)$ commute and $\theta$ is a morphism.

We will also need:

Theorem 2.3. If $\mathfrak{U}_{+}$and $\mathfrak{U}_{-}$commute with each other and if $\langle\cdot\rangle_{0}$ is $R P$, then for any $A, B, C_{i}, D_{i} \in \mathfrak{V I}_{+}$:

$$
\left|\left\langle e^{A+\theta B+\sum C_{\imath} \theta D_{\imath}}\right\rangle_{0}\right|^{2} \leqq\left\langle e^{A+\theta A+\sum C_{\imath} \theta C_{\imath}}\right\rangle_{0}\left\langle e^{B+\theta B+\sum D_{\imath} \theta\left(D_{\imath}\right)}\right\rangle_{0} .
$$

Proof. For simplicity of notation we suppose that $\mathfrak{A}$ is abelian. The general case follows by using the Trotter formula as in the proof of Theorem 2.1. Since $\langle\cdot\rangle_{0}$ is $\mathrm{RP}$, we have a Schwarz inequality $\left|\langle A \theta B\rangle_{0}\right|^{2} \leqq\langle A \theta A\rangle_{0}\langle B \theta B\rangle_{0}$ and so (here we use that $\mathfrak{U}_{+}$and $\mathfrak{H}_{-}$commute)

$$
\begin{aligned}
& \left|\left\langle A_{1} \theta\left(B_{1}\right) \ldots A_{j} \theta\left(B_{j}\right)\right\rangle_{0}\right|^{2} \\
& \leqq\left\langle A_{1} \theta\left(A_{1}\right) \ldots A_{j} \theta\left(A_{j}\right)\right\rangle_{0}\left\langle B_{1} \theta\left(B_{1}\right) \ldots B_{j} \theta\left(B_{j}\right)\right\rangle_{0} .
\end{aligned}
$$

Now

$$
\alpha \equiv e^{A+\theta B+\sum C_{\imath} \theta\left(D_{i}\right)}=e^{A} \theta\left(e^{B}\right) e^{\Sigma C_{\imath} \theta\left(D_{l}\right)},
$$

so expanding the sums we can write it as sum of terms of the form $E_{1} \theta\left(F_{1}\right) \ldots E_{l} \theta\left(F_{l}\right)$. Using $(2.2)$, we see that

$$
\left|\langle\alpha\rangle_{0}\right| \leqq \sum\left\langle\pi E_{i} \theta\left(E_{i}\right)\right\rangle_{0}^{1 / 2}\left\langle\pi F_{i} \theta\left(F_{i}\right)\right\rangle_{0}^{1 / 2},
$$

so using the Schwarz inequality for sums

$$
\left|\langle x\rangle_{0}\right|^{2} \leqq\left[\sum\left\langle\pi E_{i} \theta\left(E_{i}\right)\right\rangle_{0}\right]\left[\sum\left\langle\pi F_{i} \theta\left(F_{i}\right)\right\rangle_{0}\right] .
$$

We can now resum the exponential and so obtain the desired result.

Remarks. Notice that only (2.2) was needed to obtain the result, so we could have paralleled the discussion of GRP and given (2.2) a name. We only know how to prove (2.2) when $\mathfrak{U}_{+}$and $\mathfrak{H}_{-}$commute.

The theorems in this section are only mild abstractions of ideas in $[10,4]$. In fact, [4] already noted the importance of inequalities like those in Theorem 2.3 and of Hamiltonians of the form singled out in Theorem 2.1.

Remark. Independently, Osterwalder and Seiler have discussed RP for Euclidean Fermi lattice field theories [31] using ideas similar to ours. 
There is a generalization of Theorem 2.3, which, while it will not be used in the sequel, is potentially of interest.

Theorem 2.4. If $\mathfrak{U}_{+}$and $\mathfrak{U}_{-}$commute with each other and $\langle\cdot\rangle_{0}$ is $R P$, then for any $C_{i}, D_{i} \in \mathfrak{U}_{+}$

$$
\left|\left\langle e^{\sum_{l} C_{l} \theta D_{l}}-1\right\rangle_{0}\right|^{2} \leqq\left\langle\left(e^{\sum_{l} C_{l} \theta C_{l}}-1\right)\right\rangle_{0}\left\langle\left(e^{\sum D_{\imath} \theta D_{\imath}}-1\right)\right\rangle_{0}
$$

Proof. The same as for Theorem 2.3. One merely has to notice that the first term (namely 1) in the expansion of the exponential cancels.

Remark. Theorem 2.3 is a Corollary of Theorem 2.4. Merely add $\left(\hat{\lambda}^{-1} A+\lambda\right) \times\left(\lambda^{-1} \theta B+\lambda\right)$ to the exponential in Theorem 2.4 and then let $\lambda \rightarrow \infty$.

\section{Reflections in a Single Plane}

In this section, we consider the case where $\mathfrak{A}$ is an algebra of observables for a classical or quantum spin system on a lattice, $\langle\cdot\rangle_{0}$ is an uncoupled expectation and $\theta$ is a reflection in a plane. We concentrate on two distinct questions which are connected with our discussion in the last section: a) When is $\langle\cdot\rangle_{0} \mathrm{RP}$ and/or GRP? b) What interactions lead to a Hamiltonian with $-H=B+\theta B+\sum C_{i} \theta C_{i}$ ? We discuss the first question in a series of examples.

\section{1) Reflections in a Plane Without Sites-Classical Case}

We imagine the finite lattice $\Lambda$ (which may be a torus) being divided by a plane $\pi$ into two subsets $\Lambda_{+}$(to the "right" of $\pi$ ) and $\Lambda_{-}$, with no sites on $\pi$. There is some "reflection" $r$ on $\Lambda$ such that $r$ takes $\Lambda_{+}$into $\Lambda_{-}$and $r^{2}=1$. The "spin" at each site is a random variable taking values in a compact set $K$ with some "a priori" Borel probability distribution $d \varrho$. Let $K_{\Lambda}=\prod_{i \in \Lambda} K_{i}$ and $K_{ \pm}=\prod_{i \in \Lambda \pm} K_{i}$ (where each $K_{i}$ is a copy of $K)$. For $x \in K_{-}$, define $\theta_{*} x \in K_{+}$by $\left(\theta_{*} x\right)_{l}=x_{r(l)}$. We take $\mathfrak{A}$ to be all real-valued continuous functions on $K_{A}$ with $\mathfrak{N}_{ \pm}$the subalgebras of functions depending only on the spins in $\Lambda_{ \pm}$. Define $\theta: \mathfrak{U}_{+} \rightarrow \mathfrak{U}_{-}$by

$$
(\theta F)(x)=F\left(\theta_{*} x\right)
$$

Finally, we let $\langle F\rangle_{0}=\int_{K_{A}} F(x) \prod_{i \in \Lambda} d \varrho\left(x_{i}\right)$. Then $\langle\cdot\rangle_{0}$ is RP since

$$
\begin{aligned}
\langle F(\theta F)\rangle_{0} & =\int_{K_{-}} \int_{K_{+}} F(x) F\left(\theta_{*} y\right) \prod_{i \in \Lambda_{+}} d \varrho\left(x_{i}\right) \prod_{j \in \Lambda_{-}} d \varrho\left(y_{j}\right) \\
& =\left[\int_{K_{+}} F(x) \prod_{i \in \Lambda_{+}} d \varrho\left(x_{i}\right)\right]^{2} \geqq 0 .
\end{aligned}
$$

Since $\mathfrak{A}$ is abelian, $\langle\cdot\rangle_{0}$ is GRP. This example includes the kind of classical system in [10]. Alternatively, we could allow $\mathfrak{A}, \mathfrak{U}_{ \pm}$to be complex valued and then define $(\theta F)(x)=\overline{F\left(\theta_{*} x\right)}$. 


\section{2) Reflections in a Plane Without Sites-"Real" Quantum Case}

The setup is very similar to 1 ) but now for each $i \in \Lambda$, we take a copy $\mathscr{H}_{i}$ of $\mathbb{R}^{m}$ with the natural inner product. One defines $\mathscr{H}=\bigotimes_{i \in \Lambda} \mathscr{H}_{i}$ and $\mathscr{H}_{-}\left(\right.$resp. $\left.\mathscr{H}_{+}\right)$as the tensor product of the spaces associated with sites in $\Lambda_{-}\left(\operatorname{resp} . \Lambda_{+}\right) \cdot \mathfrak{U}$ is now all matrices on $\mathscr{H}$ and $\langle A\rangle_{0}=\operatorname{Tr}_{\mathscr{H}}(A) / \operatorname{Tr}_{\mathscr{H}}(1)$. $\mathfrak{U}_{+}$(resp. $\left.\mathfrak{A}_{-}\right)$consists of all operators of the form $1 \otimes A$ (resp. $A \otimes 1)$ under the tensor decomposition $\mathscr{H}_{=} \mathscr{H}_{-} \otimes \mathscr{H}_{+}$. Finally $\theta(1 \otimes A)=A \otimes 1$. Then for $B=1 \otimes A$

$$
\operatorname{Tr}(B \theta B)=\operatorname{Tr}_{\mathscr{H}}(A \otimes A)=\operatorname{Tr}_{\mathscr{H}}(A)^{2} \geqq 0
$$

since $\operatorname{Tr}(A)$ is real. Thus $\langle\cdot\rangle_{0}$ is RP and, since $\mathfrak{U}_{+}$and $\mathfrak{U}_{-}$commute, GRP. This example includes the quantum $x y$ model [4] in the realization $\sigma_{x}=\left(\begin{array}{ll}0 & 1 \\ 1 & 0\end{array}\right)$, $\sigma_{y}=\left(\begin{array}{rr}1 & 0 \\ 0 & -1\end{array}\right)$. Alternatively, we could take $\mathscr{H}_{i}=\mathbb{C}^{m}$ and $\theta(1 \otimes A)=\bar{A} \otimes 1$ where $^{-}$is complex conjugation.

\section{3) Reflections in a Plane Without Sites-General Quantum Case}

This is identical to the setup in (2) except for the fact that $\mathscr{H}_{i}$ is a copy of $\mathbb{C}^{m}$. If we take $\theta(1 \otimes A)=A \otimes 1$, then $\langle\cdot\rangle_{0}$ is not RP since $\operatorname{Tr}(A)$ may not be real. Indeed if $\mathfrak{U}$ and $\theta$ are chosen in some other way so that $\operatorname{Tr}$ is GRP, then the ferromagnetic Heisenberg Hamiltonian will not be expressible as $-H=B+\theta B+\sum C_{i} \theta C_{i}$, since $\operatorname{Tr}\left(\sigma_{1} \cdot \sigma_{0}\right)^{3} \leq 0$, while $\left(\sigma_{1} \cdot \sigma_{0}\right)^{3}$ is a sum of $A_{1} \theta A_{1} \ldots A_{3} \theta A_{3}$. Of course, if one takes $\theta_{1}(1 \otimes A)=\bar{A} \otimes 1$ where - is ordinary matrix complex conjugation, then for $B=1 \otimes A$

$$
\operatorname{Tr}\left(B \theta_{1} B\right)=\operatorname{Tr}_{\mathscr{H}}(\bar{A} \otimes A)=\left|\operatorname{Tr}_{\mathscr{H}+}(A)\right|^{2} \geqq 0 .
$$

So one recovers RP and GRP, but the usual Heisenberg ferromagnet is no longer of the form $\sum C_{i} \theta_{1} C_{i}$, since $\sigma_{1} \cdot \theta_{1} \sigma_{1}=\sigma_{1 x} \sigma_{0 x}+\sigma_{1 z} \sigma_{0 z}-\sigma_{1 y} \sigma_{0 y}$ in the usual realization of the $\sigma$ 's.

The fact that $\langle\cdot\rangle_{0}$ is not RP does not stop it from being RP on a subalgebra; indeed in the Heisenberg case, for functions of $\sigma_{z}$ 's alone, it is RP. It could happen that for the usual (anisotropic) Heisenberg case, $\langle\cdot\rangle_{H}$ is also RP on this subalgebra and this would lead to phase transitions in the two dimensional anisotropic case [9]. However, the failure of full GRP implies that our simple perturbation scheme of $\S 2$ will not yield a proof of this type of restricted RP.

\section{4) Twisted Reflections in a Plane Without Sites}

It is sometimes useful to define $\theta$ with a "twist". For example, in the setup of 3 ), take $m=2 S+1$ and take $\sigma_{x}, \sigma_{y}, \sigma_{z}$ as the usual spin $S$ spins; i.e. $\sigma_{z}$ is diagonal and $\sigma_{x} \pm i \sigma_{y}$ are raising and lowering operators. Thus $\sigma_{x}, \sigma_{z}$ are real and $\sigma_{y}$ is pure

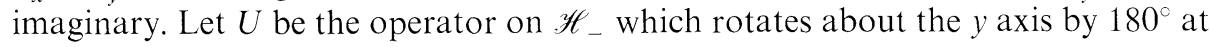
each site. Let

$$
\theta(1 \otimes A)=\overline{\left(U A U^{-1}\right)} \otimes 1 .
$$


Then for $B=1 \otimes A$

$$
\begin{aligned}
\operatorname{Tr}(B \theta B) & \left.=\operatorname{Tr} \overline{\left(U A U^{-1}\right.} \otimes A\right)=\overline{\operatorname{Tr}\left(U A U^{-1}\right)} \operatorname{Tr}(A) \\
& =|\operatorname{Tr}(A)|^{2} \geqq 0 .
\end{aligned}
$$

So $\langle\cdot\rangle_{0}$ is RP and GRP. Moreover, $\theta\left(\boldsymbol{\sigma}_{j}\right)=-\boldsymbol{\sigma}_{r(j)}$ so that the antiferromagnet $-H=-\sum_{\langle i j\rangle} \sigma_{i} \cdot \sigma_{j}$ with a sum over nearest neighbors, is of the form $B+\theta B$ $+\sum C_{i} \theta C_{i}$. This is essentially the method [4] used to discuss the antiferromagnet.

\section{5) Reflections in a Plane Containing Sites-Classical Case}

The setup is very similar to 1 ), but now there may be sites on $\pi$. Therefore we break up $\Lambda$ into three pieces, $\Lambda_{-}, \Lambda_{0}, \Lambda_{+}$corresponding to sites to the "left" of $\pi$, on $\pi$, and to the right of $\pi \cdot r$ now maps $\Lambda_{+}$to $\Lambda_{-}$and leaves $\Lambda_{0}$ invariant. $\mathfrak{H}_{+}$(resp. $\mathfrak{H}_{-}$) is the family of all functions of the spins in $\Lambda_{0} \cup \Lambda_{+}$(resp. $\Lambda_{-} \cup \Lambda_{0}$ ) and for $x=\left\{x_{i}\right\}_{i \in \Lambda-\cup \Lambda_{0}}, \theta_{*} x=x_{r(i)} \in K_{+} \times K_{0}$. As before $\langle G\rangle_{0}=\int G \prod_{i \in \Lambda} d \varrho\left(x_{i}\right)$ and $(\theta F)(x)$ $=F\left(\theta_{*} x\right)$. Then writing $(x, y, z)$ according to the decomposition $K_{-} \times K_{0} \times K_{+}$:

$$
\begin{aligned}
\langle F \theta F\rangle_{0} & =\int F(y, z) F\left(\theta_{*}(x, y)\right) \prod_{i \in \Lambda_{-}} d \varrho\left(x_{i}\right) \prod_{j \in \Lambda_{0}} d \varrho\left(y_{j}\right) \prod_{k \in \Lambda_{+}} d \varrho\left(z_{k}\right) \\
& =\int_{K_{0}} \prod_{j \in \Lambda_{0}} d \varrho\left(y_{j}\right)\left|\int F(y, z) \prod_{k \in \Lambda_{+}} d \varrho\left(z_{i}\right)\right|^{2} \geqq 0 .
\end{aligned}
$$

Thus we have RP and GRP since $\mathfrak{A}$ is abelian. This kind of reflection is mentioned in [9] and will play a major role in many of the examples in II.

\section{6) Reflections in a Plane Containing Sites-"Real" Quantum Case}

The setup is as in 2) but with the modifications in 5). Thus $\mathscr{H}=\mathscr{H}_{-} \otimes \mathscr{H}_{0} \otimes \mathscr{H}_{+}$, $\mathfrak{U}_{+}$is the linear span of the $1 \otimes A \otimes B$, and $\mathfrak{U}_{-}$the one of the $B \otimes A \otimes 1$. We take $\theta(1 \otimes A \otimes B)=B \otimes A \otimes 1$. Noticing that for $C$, an operator on $\mathscr{H}_{0} \otimes \mathscr{H}_{+}[$the analog of $(3.1)]$ :

$$
\operatorname{Tr}(C \theta C)=\operatorname{Tr}_{\mathscr{H}_{0}}\left(\left[\operatorname{Tr}_{\mathscr{H}_{+}}^{(P)}(C)\right]^{2}\right) \geqq 0,
$$

where $\operatorname{Tr}_{\mathscr{H}_{+}}^{(P)}$ is the partial trace on $\mathscr{H}_{+}$, we see that $\langle\cdot\rangle_{0}$ is RP. In this case $\mathfrak{I}_{+}$and $\mathfrak{U}_{-}$are not mutually commuting so that GRP is not automatic; indeed it is false. For let $\mathscr{H}_{+}=\mathscr{H}_{-}=\mathscr{H}_{0}=\mathbb{C}^{2}$ and let

$$
\begin{aligned}
& \theta C=\sigma_{x} \otimes\left(1+\sigma_{z}\right) \otimes 1+\sigma_{z} \otimes\left(1+\sigma_{x}\right) \otimes 1 \\
& \theta D=\sigma_{x} \otimes\left(1-\sigma_{z}\right) \otimes 1+\sigma_{z} \otimes\left(1-\sigma_{x}\right) \otimes 1
\end{aligned}
$$

in terms of the usual Pauli matrices. Then:

$$
\begin{aligned}
\operatorname{Tr}(C(\theta C) D(\theta D)) & =8 \operatorname{Tr}\left(\left(1+\sigma_{z}\right)\left(1+\sigma_{x}\right)\left(1-\sigma_{z}\right)\left(1-\sigma_{x}\right)\right) \\
& =-32<0 .
\end{aligned}
$$


Since this example is not so far from what could arise when expanding realistic spin systems, we conclude that reflections in planes containing sites are not likely to be permitted for quantum spin systems, even "real" ones.

We summarize the above examples in:

Theorem 3.1. $\langle\cdot\rangle_{0}$ is GRP for conventional reflections in planes without sites for classical and simultaneously real quantum systems and for reflections in planes with sites (lattice planes) for classical systems.

Now we turn to the question of which interactions lead to Hamiltonians of the form

$$
-H=\theta B+B+\int C(x) \theta[C(x)] d \varrho(x) .
$$

To illustrate the ideas, we will first consider the case of pair interactions in one dimension and then more general cases. The main result is that the interaction has to be "reflection positive" for (3.2) to hold. The net result of the analysis and Theorem 2.1 is that $\langle\cdot\rangle_{H}$ is RP if and only if the interaction is reflection positive. This is very reminiscent of theorems of Schoenberg [40] (see also $[2,12,38]$ ) relating positive definiteness of $e^{+t F}$ to (conditional) positive definiteness of $F$, and, indeed, our results can be viewed as a special case of that circle of ideas (see Theorem 3.5).

We begin with consideration of spins $\sigma_{-n+1}, \ldots, \sigma_{n}$.

Definition. A function $(J(j))_{j \geqq 1}$ will be called reflection positive if and only if for all positive integers $m$ and $z_{1}, \ldots, z_{m} \in \mathbb{C}$ :

$$
\sum_{i, j \geqq 1} \bar{z}_{i} z_{j} J(i+j-1) \geqq 0
$$

If we know a priori that $J$ is real-valued [it is by (3.3)] (3.3) need only be checked for $z$ real. In this case the left side of (3.3) can be viewed as the interaction between spins at sites $1, \ldots, m$ with values $z_{1}, \ldots, z_{m}$ and the reflections of these spins at $j=\frac{1}{2}$ if the basic interaction is $\sum_{\alpha, \beta} J(\alpha-\beta) \sigma_{\alpha} \sigma_{\beta}$. This explains the name given.

The following comes from the realization of (3.3) as the condition of solvability of the Hamburger moment problem. For the readers ease, we sketch a standard proof ([37]):

Proposition 3.2. Let $(J(j))_{j \geqq 1}$ be a real-valued bounded function. Then (3.3) holds if and only if

$$
J(j)=c \delta_{j 1}+\int_{-1}^{1} \lambda^{j-1} d \varrho(\lambda)
$$

for a positive measure d@ and $c \geqq 0$.

Remark. If we interpret $0^{j-1}$ as $\delta_{j 1}$, then $c \delta_{j 1}$ is just the contribution of a $\delta(\lambda)$ piece of $d \varrho$. We write it as $c \delta_{j 1}$ to be explicit.

Proof. If (3.4) holds, then

$$
\sum_{i, j \geqq 1} \bar{z}_{i} z_{j} J(i+j-1)=c\left|z_{1}\right|^{2}+\int_{-1}^{1}\left|\sum_{i=1}^{m} \lambda^{i-1} z_{i}\right|^{2} d \varrho(\lambda) \geqq 0
$$


so (3.3) holds. Conversely, if (3.3) holds, form a Hilbert space, $\mathscr{H}$, by starting with finite sequence $\left(z_{1}, \ldots, z_{m}\right)$ (arbitrary $m$ ) and letting

$$
\langle(z),(w)\rangle=\sum \bar{z}_{i} w_{j} J(i+j-1)
$$

and then dividing out by $z$ 's with $\langle(z),(z)\rangle=0$ and completing. For a finite sequence $\left(z_{1}, \ldots, z_{m}\right)$, let $A\left(z_{1}, \ldots, z_{m}\right)=\left(0, z_{1}, \ldots, z_{m}\right)$ and note that by repeated use of the Schwarz inequality:

$$
\|A z\| \leqq\|z\|^{1 / 2}\left\|A^{2} z\right\|^{1 / 2} \leqq\|z\|^{1-1 / 2^{n}}\left\|A^{2^{n}} z\right\|^{1 / 2^{n}} .
$$

But

$$
\begin{aligned}
\left\|A^{2^{n}} z\right\|^{2} & =\sum \bar{z}_{i} z_{j} J\left(i+j+2^{n+1}-1\right) \\
& \leqq\left(\sum\left|z_{i}\right|\right)^{2} \sup |J(j)|
\end{aligned}
$$

so, $\overline{\lim }\left\|A^{2^{n}} z\right\|^{1 / 2^{n}} \leqq 1$ as $n \rightarrow \infty$. We conclude that $\|A z\| \leqq\|z\|$, so $A$ extends to a map of $\mathscr{H}$ to $\mathscr{H}$. Moreover, by a direct calculation $(z, A z)=(A z, w)$. We conclude that $A$ is self-adjoint. Thus for any $z$

$$
\left(z, A^{j-1} z\right)=\int_{-1}^{1} \lambda^{j-1} d \varrho_{z}(\lambda)
$$

by the spectral theorem, where $0^{j-1}=\delta_{j 1}$. Let $z=(1,0, \ldots)$ so that $\left(z, A^{j-1} z\right)=J(j)$ and (3.4) holds.

We want to emphasize two features of (3.4). First $J \geqq 0$ is not required. Secondly only the function $J(j)=c \delta_{j 1}$ obeys (3.4) and has bounded support.

In order to obtain the simplest result relating (3.2) to (3.3) we consider free boundary conditions:

Proposition 3.3. Let $(J(j))_{j \geqq 1}$ be given. For each $m$, consider spin $1 / 2$ Ising spins, $\sigma_{-m+1}, \ldots, \sigma_{m}$ and let $\theta \sigma_{i}=\sigma_{-i+1}$

$$
-H_{m}(\sigma)=\sum_{i, j=-m+1}^{m} J(i-j) \sigma_{i} \sigma_{j} .
$$

Then $H_{m}$ has the form (3.2) for every $m$ if and only if $J$ obeys (3.3).

Remark. One half of this theorem is also contained in Hegerfeldt and Nappi [18].

Proof. If $J$ obeys (3.3), then $J$ has a representation (3.4), so that

$$
-H_{m}(\sigma)=B_{m}+\theta B_{m}+\int_{-1}^{1} C_{m}(\lambda) \theta\left[C_{m}(\lambda)\right] d \varrho(\lambda)
$$

where $B_{m}=\sum_{1<i<j<m} J(i-j) \sigma_{i} \sigma_{j}$ and $C_{m}(\lambda)=\sum_{j=1}^{m} \lambda^{j-1} \sigma_{j}$. Conversely, suppose that $H_{m}$ has the form (3.2). Then $C(x)=\sum_{i=1}^{m} \mu_{i}(x) \sigma_{i}$ and so $\int C(x)[\theta C(x)] d \varrho(x)$

$=\sum_{j \leqq 0<1 \leqq i} J(i-j) \sigma_{i} \sigma_{j}$, where, for $1 \leqq i, j \leqq m: J(i+j-1)=\int \mu_{i}(x) \mu_{j}(x) d \varrho(x)$ because if $F(\sigma)=\sum K_{i j} \sigma_{i} \sigma_{j}$, then $K_{i j}$ is unique. Thus $\sum_{1 \leqq i, j \leqq m} z_{i} \bar{z}_{j} J(i+j-1)$ $=\int\left|\sum_{i=1}^{m} z_{i} \mu_{i}(x)\right|^{2} d \varrho(x)$ and therefore $J$ is reflection positive. 
This proposition is the basic result; we present a number of extensions and variations :

A) In applications, it is useful to know that periodic boundary consitions lead to a state obeying OS positivity. Given $m$ as above, we define for $i=1,2, \ldots, 2 m-1$.

$$
J_{m}^{P}(i)=\sum_{k=-\infty}^{\infty} J(|i+2 k m|) .
$$

The Hamiltonian

$$
-H_{m}^{\mathrm{per}}=\sum_{-m+1 \leqq i<j \leqq m} J_{m}^{P}(j-i) \sigma_{j} \sigma_{i}
$$

is the Hamiltonian with periodic boundary conditions. If $J$ has the form (3.4), then

$$
J_{m}^{P}(i)=c\left[\delta_{i 1}+\delta_{i, 2 m-1}\right]+\int_{-1}^{1}\left[\lambda^{i-1}+\lambda^{-i+1} \lambda^{2 m}\right]\left(1-\lambda^{2 m}\right)^{-1} d \varrho(x)
$$

so by the above arguments, $-H=B+\theta B+\int[C(x) \theta C(x)] d_{\eta}(x)$ for suitable $C$ 's. We summarize in:

Proposition 3.4. Under the hypothesis above, if $J$ obeys (3.3), then $H_{m}^{\text {per }}$ has the form (3.2).

B) We could consider reflections about a plane containing a site. Then the above arguments imply that $J(1)$ is arbitrary and $J(i)=c \delta_{i 2}+\int_{-1}^{1} \lambda^{i-2} d \varrho(x)$ for $i \geqq 2$. In particular, in that case, one can have second "linear" neighbor coupling.

C) If one considers a multidimensional cubic system and considers reflection in the plane $i_{1}=1 / 2$, the kind of analysis above shows that what one needs is that

$$
\sum_{\imath_{1}, j_{1} \geqq 1} \bar{z}_{i} z_{j} J\left(i_{1}+j_{1}-1, i_{2}-j_{2}, \ldots, i_{v}-j_{v}\right) \geqq 0
$$

which leads to the requirement that for $i_{1} \geqq 1$

$$
J\left(i_{1}, i_{2}, \ldots, i_{v}\right)=c_{i_{2}, \ldots, i_{v}} \delta_{i_{1} 1}+\int_{-1}^{1} \lambda^{i_{1}-1} d \varrho_{i_{2}, \ldots, i_{v}}(\lambda),
$$

where $c_{i_{2}, \ldots . i_{v}}$ is a positive definite function on $\mathbb{Z}^{v-1}$ and $d \varrho$ obeys a similar condition. In particular, if

$$
\begin{aligned}
J(i) & =\alpha & \text { if } & \left|i_{1}\right|^{2}+\ldots+\left|i_{v}\right|^{2}=1 \\
& =\beta & \text { if } & \left|i_{1}\right|^{2}+\ldots+\left|i_{v}\right|^{2}=2 \\
& =0 & & \text { otherwise }
\end{aligned}
$$

(i.e. nearest neighbor coupling $\alpha$, next nearest $\beta$ ), then one will have RP about any plane bisecting a nearest neighbor bond as long as

$$
x-2|\beta|(v-1) \geqq 0 .
$$

In particular, $\beta$ can be negative. The case $\beta=-\alpha / 2(v-1)$ is of some subtlety and is discussed in detail in Paper II. [To check (3.7) is equivalent to RP, we note that the 
function $c$, which has to be positive definite on $\mathbb{Z}^{v-1}$, has a Fourier transform $c(p)=\alpha-2 \beta \sum_{j=1}^{v-1} \cos p_{j}$ so that the infimum occurs at $p_{j}=0$ (all $j$ ) if $\beta \geqq 0$ and at $p_{j}=\pi($ all $j)$ if $\beta \leqq 0$.]

D) Some clarity is obtained by considering a lattice gas in a very general language, i.e. by allowing multi-particle interactions. We will not explicitly use Theorem 2.1, and the connection with Schoenberg's work on conditionally positive definite functions will be manifest.

At each site $j \in \mathbb{Z}^{v}$ we are given a copy $K_{j}$ of some configuration space $K$ and a fixed probability measure $d \varrho\left(x_{j}\right)$ on $K_{j} ; x_{j}$ denotes a point in $K_{j}$. (For the mathematically inclined reader we remark that $K$ is assumed to be a compact Hausdorff space, and do is chosen to be a regular Borel measure. In fact all our spaces, resp. measures will have these properties.)

It helps one's intuition to imagine that $K$ is the two point set $\{1,-1\}$, and $d \varrho$ the measure assigning probability $\frac{1}{2}$ to 1 and -1 . This will correspond to Ising models (see also Corollary 3.6, below).

Given a subset $X \subseteq \mathbb{Z}^{\prime}$, we define

$$
K^{X}=\chi_{j \in X} K_{j} \quad \text { and } \quad K^{\infty}=K^{\mathbb{Z}^{v}}
$$

(Since $K$ is a compact Hausdorff space, so is $K^{X}$, for all $X \subseteq \mathbb{Z}^{v}$.)

To each bounded subset $\Lambda \subset \mathbb{Z}^{v}$ there corresponds a finite system in $\Lambda$ with configuration space $K^{\Lambda}$, an algebra of "observables" $C\left(K^{\Lambda}\right)$, and whose states are the probability measures on $K^{\Lambda}$. [These are precisely the continuous, normalized, positive linear functionals on $C\left(K^{\Lambda}\right)$.]

We denote by tr the expectation on $C\left(K^{\infty}\right)$ given by the product measure $\prod_{j \in \mathbb{Z}^{v}} d \varrho\left(x_{j}\right)$. Clearly tr defines a state of the finite system in $\Lambda$, denoted $\operatorname{tr}_{\Lambda}$, by restriction to $C\left(K^{\Lambda}\right)$.

The dynamics of such systems is given in terms of an interaction, $\Phi$. This is a map from bounded subsets $X \subset \mathbb{Z}^{v}$ to $C\left(K^{\infty}\right)$ with the properties that

$$
\Phi(X) \in C\left(K^{X}\right),
$$

and

$$
\operatorname{tr}_{Y}(\Phi(X))=\int \prod_{j \in Y} d \varrho\left(x_{j}\right) \Phi(X)(x)=0,
$$

for all $Y$ with $Y \cap X \neq \emptyset ; x=\left\{x_{j}\right\}_{j \in \mathbb{Z}^{v}}$.

Condition (3.9) is not loss of generality: given an arbitrary interaction $\tilde{\Phi}$ satisfying (3.8), one can always find a physically equivalent interaction $\Phi$ obeying (3.8) and (3.9)!

The Hamilton function of a finite system in $\Lambda$ with interaction $\Phi$ is given by

$$
H_{A}^{\Phi}=\sum_{X \subset A} \Phi(X)
$$


and the Gibbs equilibrium state with boundary condition $\varrho_{c \Lambda} \in L^{1}\left(K^{\Lambda}, \prod_{j \in \Lambda} d \varrho\left(x_{j}\right)\right)$, describing the interactions of the system in $\Lambda$ with its complement in $\Lambda^{c}$ (recall the Dobrushin-Lanford-Ruelle equations [39,22]), is given by

$$
\langle F\rangle\left(\Phi, \varrho_{i A}\right)=Z_{\Lambda}^{-1} \operatorname{tr}_{\Lambda}\left(\mathrm{Fe}^{-H_{A}^{\Phi}} \varrho_{i \Lambda}\right),
$$

for arbitrary $F \in C\left(K^{\Lambda}\right)$. Here

$$
Z_{\Lambda}=\operatorname{tr}_{\Lambda}\left(e^{-H_{\Lambda}^{\Phi}} \varrho_{\hat{C} \Lambda}\right) \text {. }
$$

We now consider a decomposition of $\mathbb{Z}^{v}$ into two disjoint sublattices $\Gamma_{+}, \Gamma_{-}$ (generally separated by a hyperplane); $r$ is the reflection taking $\Gamma_{-}$to $\Gamma_{+}$and $\theta_{*}$ the obvious reflection map from $K^{\Gamma_{-}}$to $K^{\Gamma_{+}}$. For $F \in C\left(K^{\Gamma_{+}}\right)$, we set

$$
\theta F\left(x_{-}\right)=\overline{F\left(\theta_{*} x_{-}\right)},
$$

where $x_{ \pm}=\left\{x_{j}\right\}_{j \in \Gamma_{ \pm}}$; we set $\Lambda_{ \pm}=\Lambda \cap \Gamma_{ \pm}$, and if $\Lambda_{+}=r \Lambda_{-}$we say that $\Lambda$ is reflection symmetric (RS).

Our previous notion of RP is equivalent to

$$
\langle F \theta F\rangle\left(\Phi, \varrho_{\hat{c} \Lambda}\right) \geqq 0,
$$

for all $F \in C\left(K^{\Lambda+}\right)$. In this case $\langle-\rangle\left(\Phi, \varrho_{\hat{i} A}\right)$ is said to be RP.

We say that a b.c. $\varrho_{\partial \Lambda}$ satisfies RP iff $\operatorname{tr}_{\Lambda}\left(F \theta F \varrho_{\hat{C} \Lambda}\right) \geqq 0$, for all $F \in C\left(K^{\Lambda+}\right)$.

Clearly there are b.c. $\varrho_{\delta A}$ which are not RP, but there are also plenty of b.c. which are (e.g. $\varrho_{\partial \Lambda}=\sum_{k} G_{k} \theta G_{k}, G_{k} \in C\left(K^{\Lambda^{+}}\right)$for all $\left.k\right)$ !

Remark. Consider two b.c. $\varrho_{\hat{c} \Lambda}$ and $\varrho_{\partial A}^{\prime}$ such that

$$
\varrho_{\hat{c} \Lambda} \cdot \varrho_{\partial \Lambda}^{\prime} \in L^{1}\left(K^{\Lambda}, \prod_{j \in \Lambda} d \varrho\left(x_{j}\right)\right) .
$$

If $\varrho_{\partial \Lambda}$ and $\varrho_{\partial \Lambda}^{\prime}$ are RP then so is

$$
\varrho_{\partial A}^{\prime \prime}=\varrho_{\hat{\delta} A} \cdot \varrho_{\partial A}^{\prime},
$$

by Schur's theorem.

From now on we shall always assume that $\Phi$ is reflection covariant, i.e.

$$
\theta \Phi(X)=\Phi(r X)
$$

for arbitrary $X \subset \Gamma_{+}$.

Our aim is to state and prove a necessary and sufficient condition on an interaction $\Phi$ such that $\langle-\rangle\left(\Phi, \varrho_{\hat{\sigma} \Lambda}\right)$ is RP, for all RP b.c. $\varrho_{\hat{\delta} \Lambda}$ and all bounded, RS regions $\Lambda$. if

We call an interaction CRN (for "conditionally reflection negative") if and only

$$
\sum_{X \cap \Gamma_{ \pm} \neq \emptyset} \operatorname{tr}(F \theta F \Phi(X)) \leqq 0,
$$

for all $F \in C\left(K^{Y}\right)$, with $Y$ an arbitrary bounded subset of $\Gamma_{+}$, obeying $\operatorname{tr}(F)=0$. 
We call an interaction $\Phi$ RN (for "reflection negative") if and only if

$$
\sum_{X \cap \Gamma_{ \pm} \neq \vartheta} \operatorname{tr}(F \theta F \theta(X)) \leqq 0,
$$

for all $F \in C\left(K^{Y}\right)$ and for arbitrary, bounded $Y \subset \Gamma_{+}$.

Let $\operatorname{diam} X=\max \{|i-j|: i, j \in X\}$, let $X+a$ denote the translate of $X$ by a vector $a \in \mathbb{Z}^{v}$, and let $\tau_{a}$ denote the natural isomorphism from $C\left(K^{X}\right)$ to $C\left(K^{X+a}\right)$, for arbitrary $X$, i.e. $\left\{\tau_{a}\right\}$ are the translations. Finally, let $\|\cdot\|$ denote the supnorm on $C\left(K^{\infty}\right)$.

Theorem 3.5. 1) The Gibbs state $\langle-\rangle\left(\beta \Phi, \varrho_{i \Lambda}\right)$ is RP, for all inverse temperatures $\beta \geqq 0$, all RP b.c. $\varrho_{i \Lambda}$ and all RS regions $\Lambda$ if and only if $\Phi$ is CRN.

2) Suppose an interaction $\Phi$ fulfills (3.9) and has the property that

$\sup \{\|\Phi(X)\|: \operatorname{diam} X \geqq r\} \rightarrow 0$,

as $r \rightarrow \infty$ (this condition is fulfilled if $\Phi$ obeys any reasonable condition of thermodynamic stability!) Then $\Phi$ is CRN if and only if $\Phi$ is RN.

3) If $\Phi$ is RN and $A$ some $\mathrm{RS}$ bounded set then

$$
\sum_{X \cap A_{ \pm} \neq \emptyset} \Phi(X)
$$

is a weak limit of functions of the form

$$
-\sum_{k} G_{k}^{A} \theta G_{k}^{A}
$$

where $G_{k}^{A} \in C\left(K^{\Lambda+}\right)$, for all $k$. An analogous statement holds for RP b.c. $\varrho_{\hat{c} \Lambda}$.

Remarks. 1) The class of (C)RN interactions $\Phi$ forms a convex cone. An analogous statement holds for RP b.c. By (3.13), the convex cone of RP b.c. is multiplicative. Furthermore, note that RP is stable under taking the thermodynamic limit $\Lambda \uparrow \mathbb{Z}^{v}$ through a sequence of RS regions $\Lambda$, with RP b.c. $\varrho_{\hat{\sigma} \Lambda}$.

These facts and Theorem 3.5 represent a rather complete, mathematical characterization of RP Gibbs states in the classical case; see also Corollary 3.6.

2) Generally, CRN interactions and periodic b.c. lead to RP Gibbs states; (see also Proposition 3.4). If $\Phi$ obeys (3.17) and the periodic Gibbs states are RP, for all bounded hyper cubes $\Lambda$, then $\Phi$ must be RN.

Clearly, periodic b.c. lead to translation invariance, so that $\Lambda$ is $\mathrm{RS}$ with respect to many different pairs of hyperplanes, and - if $\Phi(X+a)=\tau_{a}(\Phi(X))$ (translation invariance) - the Gibbs state is translation invariant. For these reasons translation invariant $\Phi$ 's and periodic b.c. play an (annoyingly) important role in our theory. Proof of Theorem 3.5. 1) First we choose $\varrho_{\hat{c} A}=1$. This b.c. is clearly RP. In this case, the Gibbs state $\langle-\rangle(\beta \Phi, 1)$ is RP if and only if

$$
R_{\Lambda}^{\beta \Phi}=\exp \left[-\sum_{\substack{X \subset A \\ X \cap \Gamma_{+} \neq \emptyset}} \beta \Phi(X)\right]
$$

has the property

$$
\operatorname{tr}\left(F \theta F R_{A}^{\beta \Phi}\right) \geqq 0,
$$


for all $F \in C\left(K^{\Lambda+}\right)$. This follows easily from (3.14) and the definition of the Gibbs state. If $R_{\Lambda}^{\beta \Phi}\left(x_{+}, x_{-}\right)$denotes the integral kernel of $R_{\Lambda}^{\beta \Phi}$ the above inequality takes the form

$$
\int \prod_{j \in I_{+}} d \varrho\left(x_{j}\right) d \varrho\left(y_{j}\right) F\left(x_{+}\right) \overline{F\left(y_{+}\right)} R_{\Lambda}^{\beta \Phi}\left(x_{+}, \theta_{*} y_{+}\right) \geqq 0
$$

for all $F \in C\left(K^{\Lambda_{+}}\right)$.

Assuming that (3.18) holds for arbitrary RS regions $\Lambda$ and all $\beta \geqq 0$ and using a straight forward extension of Schoenberg's theorem [38] (Theorem XIII.52) we conclude that $\Phi$ must be CRN, i.e.

$$
\sum_{X \cap \Gamma_{ \pm} \neq \emptyset} \operatorname{tr}(F \theta F \Phi(X)) \leqq 0
$$

for all $F \in C\left(K^{\Lambda_{+}}\right)$with $\operatorname{tr}(F)=0$ and arbitrary, bounded $\Lambda_{+} \subset \Gamma_{+}$. [Here we have used (3.9) to include regions $X \not \subset \Lambda$ in the summation. We recall that Schoenberg's theorem says that a matrix $\left(b_{i j}\right)$ has the property that $\left(e^{\beta b_{\imath \jmath}}\right)$ is positive definite for all $\beta \geqq 0$ if and only if $\sum \bar{z}_{i} z_{j} b_{i j} \geqq 0$ for all $z$ 's with $\sum z_{i}=0$.] This proves one direction of Theorem 3.5(1). Conversely suppose now that $\Phi$ is CRN. Then $\sum_{X \cap \Gamma_{ \pm} \neq \emptyset} \operatorname{tr}(F \theta F \Phi(X)) \leqq 0$, for all $F \in C\left(K^{\Lambda_{+}}\right)$with $\operatorname{tr}(F)=0$, for any RS region $\Lambda$. Now fix some RS, bounded $\Lambda$. By (3.9), it follows that

$$
\sum_{X \cap A_{ \pm} \neq \theta} \operatorname{tr}(F \theta F \Phi(X))=\sum_{\substack{X \cap A_{ \pm} \neq \vartheta \\ X \subseteq A}} \operatorname{tr}_{A}(F \theta F \Phi(X)) \leqq 0,
$$

for all $F \in C\left(K^{\Lambda_{+}}\right)$with $\operatorname{tr}_{\Lambda}(F)=0$. If we write this out as an integral and use Schoenberg's theorem in the other direction we immediately conclude that $R_{A}^{\beta \Phi}\left(x_{+}, \theta_{*} y_{+}\right)$is a positive definite kernel.

Next, if $\varrho_{\partial A}$ is RP then the kernel of $\varrho_{\delta A}, \varrho_{\partial A}\left(x_{+}, \theta_{*} y_{+}\right)$is positive definite. By Schur's theorem, $R_{\Lambda}^{\beta \Phi}\left(x_{+}, \theta_{*} y_{+}\right) \varrho_{\hat{c} \Lambda}\left(x_{+}, \theta_{*} y_{+}\right)$is positive definite, so that

$$
\begin{aligned}
\int \prod_{j \in \Lambda_{+}} d \varrho\left(x_{j}\right) d \varrho\left(y_{j}\right) F\left(x_{+}\right) \overline{F\left(y_{+}\right)} R_{\Lambda}^{\beta \Phi}\left(x_{+}, \theta_{*} y_{+}\right) \varrho_{\hat{c} \Lambda}\left(x_{+}, \theta_{*} y_{+}\right) \\
\quad=\operatorname{tr}_{\Lambda}\left(F \theta F R_{\Lambda}^{\beta \Phi} \varrho_{\partial \Lambda}\right) \geqq 0
\end{aligned}
$$

for all $F \in C\left(K^{\Lambda_{+}}\right)$.

Since, by condition (3.14), $e^{-\beta H_{A}^{\Phi}}=e^{-H_{A}^{\beta \Phi}}$ is obviously of the form $G^{\Lambda} \theta G^{\Lambda} R_{\Lambda}^{\beta \Phi}$, with $G^{\Lambda} \in C\left(K^{\Lambda_{+}}\right)$, Theorem 3.5.1) is now proven.

2) It is trivial that if $\Phi$ is RN then $\Phi$ is CRN. Therefore we must only show that if $\Phi$ is CRN and satisfies (3.9) and (3.17) then $\Phi$ is RN. For this purpose, let $F \in C\left(K^{Y}\right)$, for an arbitrary, but hence forth fixed $Y \subset \Gamma_{+}$. We define

$$
G=F-\tau_{a}(F),
$$


where a is a translation such that $Y+a \subset \Gamma_{+}$, i.e. $G \in C\left(K^{Y \cup Y+a}\right)$ with $Y \cup Y+a \subset \Gamma_{+}$. Clearly $\operatorname{tr}(G)=\operatorname{tr}(F)-\operatorname{tr}\left(\tau_{a}(F)\right)=\operatorname{tr}(F)-\operatorname{tr}(F)=0$. Hence if $\Phi$ is CRN then

$$
\begin{aligned}
& \sum_{X \cap \Gamma_{ \pm} \neq 0} \operatorname{tr}(G \theta G \Phi(X)) \leqq 0, \quad \text { i.e. } \\
& \sum_{X \cap \Gamma_{ \pm} \neq \emptyset} \operatorname{tr}(F \theta F \Phi(X))-\sum_{X 1 \cap \Gamma_{ \pm} \neq \emptyset} \operatorname{tr}\left(F \theta \tau_{a}(F) \Phi\left(X_{1}\right)\right) \\
& -\sum_{X_{2} \cap \Gamma_{\text {土 }} \neq \emptyset} \operatorname{tr}\left(\tau_{a}(F) \theta F \Phi\left(X_{2}\right)\right) \\
& +\sum_{X_{3} \cap \Gamma_{ \pm} \neq \emptyset} \operatorname{tr}\left(\tau_{a}(F) \theta \tau_{a}(F) \Phi\left(X_{3}\right)\right) \leqq 0 .
\end{aligned}
$$

By condition (3.9), the only non-vanishing terms in the last three sums on the l.s. of this inequality fulfill the conditions $X_{1} \subset Y \cup r(Y+a), X_{2} \subset Y+a \cup r Y$ and $X_{3} \subset(Y+a) \cup r(Y+a)$. Moreover $X_{j} \cap \Gamma_{ \pm} \neq \emptyset, j=1,2,3$. Applying now condition (3.17) we see that these three sums thend to 0 as a tends to $\infty$ in a direction for which $I_{+}+a \subset \Gamma_{+}$, for all a of this direction. Thus

$$
\sum_{X \cap \Gamma_{ \pm} \neq 0} \operatorname{tr}(F \theta F \Phi(X)) \leqq 0
$$

for all $F \in C\left(K^{Y}\right)$. Since $Y$ is an arbitrary, bounded set in $\Gamma_{+}$, this proves Theorem $3.5(2)$.

3) Let $P$ be an orthogonal projection on $L_{+}^{2}=L^{2}\left(K^{\Lambda_{+}}, \sum_{j \in \Lambda_{+}} d \varrho\left(x_{j}\right)\right)$. Then the distribution kernel of $P, P\left(x_{+}, y_{+}\right)$, is a weak limit of functions of the form

$\sum_{k} \Psi_{k}\left(x_{+}\right) \overline{\Psi_{k}\left(y_{+}\right)}$, where $\Psi_{k} \in L_{+}^{2}, \quad$ for all $k$.

This observation combined with the spectral theorem for negative, (resp. positive) bounded operators and the relation $\overline{\Psi_{k}\left(\theta_{*} y_{-}\right)}=\left(\theta \Psi_{k}\right)\left(y_{-}\right)$clearly proves Theorem $3.5(3)$.

As an application of this general theory we consider a classical spin system with many body interactions. The classical spin at site $i$ is denoted $\sigma_{i}$, and $\sigma_{X}$ $=\prod_{i \in X} \sigma_{i}$. The expectation $\operatorname{tr}$ is chosen such that $\operatorname{tr}\left(\sigma_{X}\right)=0$ and $\operatorname{tr}\left(\sigma_{X}^{2}\right)>0$, for all nonempty $X$. The interaction $\Phi$ is given by

$$
\Phi: X \rightarrow-J_{X} \sigma_{X}
$$

where $J=\left\{J_{X}\right\}$ is a family of real numbers indexed by the bounded subsets of $\mathbb{Z}^{v}$. The interaction $\Phi$ is translation invariant if $J_{X+a}=J_{X}$, for all $a \in \mathbb{Z}^{v}$, and reflection covariant, see (3.14), if $J_{X}=J_{r X}$, for all $X \subset \Gamma_{+}$.

Example. Ising model with multi-spin interactions.

Definition. We say that $J$ is RP if and only if

$$
\sum_{X . Y \subset A_{+}} \bar{z}_{X} z_{Y} J_{X \cup r Y} \geqq 0,
$$

for arbitrary, finite sequences $\left\{z_{X}\right\}_{X \subset \Gamma_{+}}$of complex numbers. 
Corollary 3.6. 1) Let $\Phi$ be given by (3.19). Then $\Phi$ is $C R N$ if and only if $J$ is $R P$.

2) The family of all RP J's forms a convex, multiplicative cone.

Proof. 1) It is not hard to see that if $J$ is RP then $\Phi$, given by (3.19), is RN, thus CRN. Conversely, if $\Phi$ is CRN then, for an arbitrary function $F$ of $\left\{\sigma_{j}\right\}_{j \in \Gamma_{+}}$with $\operatorname{tr}(F)=0$

$\sum_{X, Y \subset \Gamma_{+}} J_{X \cup r Y} \overline{\operatorname{tr}\left(F \sigma_{X}\right)} \operatorname{tr}\left(F \sigma_{Y}\right) \geqq 0$.

Now choose $F=\sum \tilde{z}_{X} \sigma_{X}$, where $\tilde{z}_{X}=z_{X} \operatorname{tr}\left(\sigma_{X}^{2}\right)^{-1}$, and $\left\{z_{X}\right\}_{X \subset \Gamma_{+}}$is a finite sequence of complex numbers. Then

$$
\operatorname{tr}(F)=\sum \tilde{z}_{X} \operatorname{tr}\left(\sigma_{X}\right)=0
$$

and

$\operatorname{tr}\left(F \sigma_{X}\right)=\sum_{Y} \tilde{z}_{Y} \operatorname{tr}\left(\sigma_{Y} \sigma_{X}\right)=\sum_{Y} \tilde{z}_{Y} \operatorname{tr}\left(\sigma_{Y \cap X}^{2}\right) \operatorname{tr}\left(\sigma_{Y \Delta X}\right)=\tilde{z}_{X} \operatorname{tr}\left(\sigma_{X}^{2}\right)=z_{X}$,

so

$$
\sum_{X, Y \subset \Gamma_{+}} J_{X \cup r Y} \overline{\operatorname{tr}\left(F \sigma_{X}\right)} \operatorname{tr}\left(F \sigma_{Y}\right)=\sum_{X, Y \subset \Gamma_{+}} \bar{z}_{X} z_{Y} J_{X \cup r Y},
$$

and, by (3.21) and (3.22), this is non-negative. Since $\left\{z_{X}\right\}$ is arbitrary, it follows that $J$ is RP.

2) Convexity is obvious. Given $J$ and $J^{\prime}$, both RP, we define $J^{\prime \prime}$ by

$J_{X}^{\prime \prime}=J_{X} \cdot J_{X}^{\prime}$, for all $X$.

By Schur's theorem $J_{X}^{\prime \prime}$ is then also RP.

Remark. There are plenty of RP $J$ 's with the property that $J_{X} \neq 0$, for subsets $X$ containing an arbitrarly large number of sites. (As an excercise we recommend that the reader construct some explicit examples of this type.) As a largely open problem we propose to investigate the detailed geometric properties of the cone of RN interaction within one of the standard Banach spaces of interactions, [39].

Theorem 3.5 and Corollary 3.6 provide a rather satisfactory, general theory of RP Gibbs states for classical systems. See also [6]. In the quantum case no complete characterization of RP Gibbs states is available, yet.

The reader can check that Theorem 3.5/Corollary 3.6 includes results in Proposition 3.3 and its consequences via Theorem 2.1 as a special case. In particular, the following should be noted. In Proposition 3.3, we assumed that $H$ has the form (3.2). This form was chosen so that the Gibbs state $\langle\cdot\rangle_{\beta H}$ is RP for all $\beta$. If, instead, one starts with the apparently weaker requirement that $\langle\cdot\rangle_{\beta H}$ is RP for all $\beta$, then Theorem 3.5.3) tells us that $H$ has to be of the form (3.2).

Example. Consider a two-dimensional Ising model with 2, 3, and 4 body interactions. Let

$$
\begin{aligned}
& X=\sigma_{(0,0)} \sigma_{(0,1)} \sigma_{(1,1)} \sigma_{(1,0)}, \\
& Y=\sigma_{(0,0)} \sigma_{(1,0)}\left[\sigma_{(1,1)}+\sigma_{(0,1)}\right], \\
& Z=\sigma_{(0,0)} \sigma_{(1,1)} .
\end{aligned}
$$


Let $-H=\sum_{a \in A} \tau_{a}[J X+K Y+L Z]$ where $J, K, L$ are numbers and $\tau_{a}$ represents translation by a unit. $H$ will be RN with reflection about the plane $i_{1}=1 / 2$ if $K^{2}=J L$ and $J, L \geqq 0$. To see this, note that in this case $-H$ has the form $B+\theta B+\sum_{i} C_{i} \theta C_{i}$, where $C \alpha \sigma_{(1,0)} \sigma_{(1,1)}+\beta \sigma_{(1,0)}$ and hence $C \theta C=\alpha^{2} X+\alpha \beta Y$ $\left.+\beta^{2} Z\right]$, and the sum on $i$ is over translations in the plane $i_{1}=1 / 2$.

\section{Chessboard Estimates and Infrared Domination}

In this section, we review, systematize and extend the basic methods of $[10,4,14$, 9] which are based on the use of RP about a large number of planes. For this reason, we will have to work with periodic boundary conditions or directly in infinite volume. We begin by describing "chessboard estimates", then mention the way these can be used in connection with a Peierls argument, and finally discuss the method of infrared bounds.

Theorem 4.1. (Abstract Chessboard Estimates [9]). Let $\mathfrak{H}_{0}$ be a real vector space, let $r: \mathfrak{U}_{0} \rightarrow \mathfrak{U}_{0}$ be a real linear map with $r^{2}=1$ and let $F\left(a_{1}, \ldots, a_{2 n}\right)$ be a complexvalued multilinear map obeying:

$$
F\left(a_{1}, \ldots, a_{2 n}\right)=F\left(a_{2}, \ldots, a_{2 n}, a_{1}\right)
$$

and

$$
\begin{aligned}
& \left|F\left(a_{1}, \ldots, a_{n}, b_{n}, \ldots, b_{1}\right)\right|^{2} \\
& \quad \leqq F\left(a_{1}, \ldots, a_{n}, r a_{n}, \ldots, r a_{1}\right) F\left(b_{1}, \ldots, b_{n}, r b_{n}, \ldots, r b_{1}\right) .
\end{aligned}
$$

Then $\|a\| \equiv|F(a, r a, a, \ldots, r a)|^{1 / 2 n}$ is a semi-norm and

$$
\left|F\left(a_{1}, \ldots, a_{2 n}\right)\right| \leqq \prod_{i=1}^{2 n}\left\|a_{i}\right\| .
$$

Remarks. 1. In the example of $2 n$ spins on a line, one should think of $\mathfrak{A}_{0}$ as functions of a spin ata single site, and $F\left(a_{1}, \ldots, a_{2 n}\right)=\left\langle\prod_{i=-n+1}^{n} a_{i+n}\left(\sigma_{i}\right)\right\rangle ; r(a)=a$ (or $\bar{a}$ if we take complex valued functions) so that (4.1) is true if periodic boundary conditions are used and (4.2) is an expression of RP.

2. The statement and proof are patterned on [9]. For a discussion of its field theory forebears see [43]. For applications to Hölder's inequality for matrices, see [6].

3. It is a worthwhile exercise to prove this directly for the case $2 n=4$, see $[6,43]$.

4. By (4.2) the $F\left(a_{1}, \ldots, a_{n}, r a_{n}, \ldots, r a_{1}\right)$ are either all $\geqq 0$ or all $\leqq 0$. We can suppose the former without loss.

Proof. We first prove (4.3) and then it follows that $\|\cdot\|$ is a semi-norm, since (4.3) implies the triangle inequality. Let $a_{1}, \ldots, a_{2 n}$ be given and suppose that $\left\|a_{i}\right\| \neq 0$ for all $i$. Let $b_{1}, \ldots, b_{2 n}$ be any $2 n$ elements each of which is either an $a_{i}$ or an $r\left(a_{i}\right)$. Let

$$
g\left(b_{1}, \ldots, b_{2 n}\right) \equiv F\left(b_{1}, \ldots, b_{2 n}\right) / \prod_{i=1}^{2 n}\left\|b_{i}\right\|
$$


and let $g_{0}=\max \left|g\left(b_{i}\right)\right|$ as the $b_{i}$ run through the $(4 n)^{2 n}$ possibilities. Among all choices with $\left|g\left(b_{i}\right)\right|=g_{0}$, pick one with the longest string of the form $a_{i}, r\left(a_{i}\right)$, $a_{i}, \ldots, r\left(a_{i}\right)$ for $b_{1}, \ldots, b_{2 l}$. Since (4.1) implies that $\left\|r\left(a_{i}\right)\right\|=\left\|a_{i}\right\|$, (4.2) shows that $g$ obeys the same Schwarz inequality as $F$. Thus, if $\left|g\left(b_{1}, \ldots, b_{2 n}\right)\right|=g_{0}$, we must have that $\left|g\left(b_{1}, \ldots, b_{n}, r b_{n}, \ldots, r b_{1}\right)\right|=g_{0}$. If $2 l$ is not $2 n$ in the above choice, let $b_{1}^{\prime}, \ldots, b_{2 n}^{\prime}$ be a cyclic permutation of $b_{1}, \ldots, b_{2 n}$ with $a_{i}, r\left(a_{i}\right), \ldots, a_{i}, r\left(a_{\imath}\right)$ occuring as $b_{n-j}^{\prime}, \ldots, b_{n}^{\prime}$ where $j=n-1$ if $2 l>n$ and otherwise $j=2 l-1$. But then $b_{1}, \ldots, b_{n}, r b_{n}, \ldots, r b_{1}$ has a string of the form $a_{i}, r\left(a_{i}\right), \ldots$ of length $2 j+2$. It follows that $g_{0}=\left|g\left(a_{i}, r\left(a_{i}\right), \ldots, r\left(a_{i}\right)\right)\right|$ for some $a_{l}$. But such a $g$ is always 1 so $g_{0} \leqq 1$. This implies (4.3) if each $\left\|a_{i}\right\| \neq 0$.

If some $\left\|a_{i}\right\|=0$, we claim that $F\left(a_{i}\right)=0$. For, if not, let $b_{1}, \ldots, b_{2 n}$ be a sequence with some $b_{j}=a_{i}$ so that the longest string $a_{i}, r\left(a_{i}\right), \ldots, r\left(a_{i}\right)$ occurs consistent with $F\left(b_{j}\right) \neq 0$. As above $b_{1}, \ldots, b_{2 n}$ must be $a_{v}, r\left(a_{i}\right), \ldots, r\left(a_{i}\right)$ so there is a contradiction.

Typical of the explicit versions of Theorem 4.1 are the following:

Theorem 4.2. Let $\Lambda$ be a rectangular subset of $\mathbb{Z}^{v}$ with sides $2 n_{1} \times \ldots \times 2 n_{v}$ $\left(n_{1}, \ldots, n_{v}\right.$ positive integers). Let $\langle\cdot\rangle$ be an expectation value for a classical spin system which is invariant under translations $\bmod n_{i}$ (periodic boundary conditions) and which is RP with respect to (untwisted) reflections $\left(\bmod n_{i}\right)$ in all planes perpendicular to coordinate axes running mid-way between neighboring points of $A$. Then for any functions $\left\{G_{\alpha}\right\}_{x \in A}$ :

$$
\left|\left\langle\prod_{\gamma \in \Lambda} G_{\alpha}\left(\sigma_{\alpha}\right)\right\rangle\right| \leqq \prod_{\alpha \in \Lambda}\left\langle\prod_{\beta \in \Lambda} G_{\alpha}\left(\sigma_{\beta}\right)\right\rangle^{1 /|\Lambda|}
$$

Proof. Let $\mathfrak{U}_{0}$ be the functions of spins $\left\{\sigma_{x}\right\}_{\alpha \in \Lambda ; \alpha_{1}=1}$ and let

$$
F\left(a_{1}, \ldots, a_{2 n_{1}}\right)=\left\langle\prod_{j=1}^{2 n_{1}} a_{j}\left(\left\{\sigma_{\alpha}\right\}_{\alpha_{1}=j}\right)\right\rangle .
$$

Using the assumed RP and Theorem 4.1, and setting $a_{j}=\prod_{\alpha_{2}, \ldots, \alpha_{v}} G_{J, \alpha_{2}, \ldots . \alpha_{v}}$, we
obtain

$$
\left|\left\langle\prod_{x \in \Lambda} G_{\gamma}\left(\sigma_{\alpha}\right)\right\rangle\right| \leqq \prod_{j=1}^{2 n_{1}}\left\langle\prod_{k=1}^{2 n_{1}} \prod_{\alpha_{2}, \ldots, \alpha_{v}} G_{j, \alpha_{2}, \ldots, \alpha_{v}}\left(\sigma_{k, \alpha_{2}, \ldots, \alpha_{v}}\right)\right\rangle^{1 / 2 n_{1}} .
$$

Repeating the argument in the other $v-1$ directions, (4.4) results.

Now let $j$ be an element of the dual lattice, $\tilde{\Lambda}$, to $\Lambda$, i.e. $j$ is the center of a unit cube, $\Delta_{j}$ contained in $\Lambda$. Let $F$ be a function of the spins in $\Lambda$. We say that $F \in \Sigma_{j}$ if and only if $F$ is only a function of spins at the corners of $\Delta_{j}$. Given such an $F$ we set

$$
\gamma(F)=\left\langle\prod_{i \in \Lambda} \tilde{F}_{(i)}\right\rangle^{1 /|\Lambda|}
$$

where $\tilde{F}_{(i)}$ is $F$ for $i=j$ and for nearest neighbor cubes $\Delta_{i}$ and $\Delta_{i^{\prime}}, \tilde{F}_{(i)}=\theta_{i i^{\prime}}\left[\tilde{F}_{(i)}\right]$ with $\theta_{i i^{\prime}}$ untwisted reflection in the plane separating $\Delta_{i}$ and $\Delta_{i^{\prime}}$. Thus, if $i-j$ has all even components, then $F_{(i)}$ is a translate of $F$ and if $i-j$ has $v_{0}$ odd components $F$ is a translate of $F$ reflected in $v_{0}$ orthogonal planes. The proof of Theorem 4.2 extends to: 
Theorem 4.3. If $\Lambda$ is the set in Theorem $4.2,\langle\cdot\rangle$ is translation invariant and $R P$ with respect to planes perpendicular to the coordinate axes but through the sites then

$$
\left\langle\prod_{i \in \Lambda} F_{i}\right\rangle \leqq \prod_{i \in \Lambda} \gamma\left(F_{i}\right)
$$

for $F_{i} \in \sum_{i}$.

There are clearly quantum variants and variants with various oblique planes. Except for some discussion of the face centered cubic lattice at the close of this section we do not make these explicit. Reflections at oblique planes have also been used in $[41,17]$.

To explain schematically the Peierls-chessboard method, consider a classical spin system and break up the configuration space $K$ into pieces $K_{1} \cup \ldots \cup K_{m}$. (For example, if $K$ is finite, each $K_{j}$ could be a single point. For the anisotropic classical Heisenberg model, $K=$ unit sphere, and $K_{1}$ and $K_{2}$ are the two "polar caps" of the sphere, and $K_{3}$ is the temperate and tropical regions.) Let $P_{\alpha}^{(j)}$ be the function which is 1 (resp. 0) if $\sigma_{\alpha}$ is in $K_{j}$ (resp. not in $K_{j}$ ). Let $\langle A\rangle_{\beta, A}$ $=\left\langle A e^{-\beta H_{A}}\right\rangle_{0} /\left\langle e^{-\beta H_{\Lambda}}\right\rangle_{0}$ where $\beta>0$ and $H_{\Lambda}$ is the Hamiltonian for the lattice $\Lambda$. Let $\langle\cdot\rangle_{\beta, \infty}$ be some weak-* limit point of $\langle\cdot\rangle_{\beta, \Lambda}$ as $\Lambda \rightarrow \mathbb{Z}^{v}$. As we will describe, the Peierls-chessboard method typically allows one to show that for $i \neq j$, $\left\langle P_{\alpha}^{(i)} P_{\gamma}^{(j)}\right\rangle_{\beta, \Lambda} \rightarrow 0$ as $\beta \rightarrow \infty$ uniformly in $\Lambda, \alpha, \gamma$. Suppose that we also know that for $i=1,2, \lim _{\beta \rightarrow \infty}\left\langle P_{\alpha}^{(i)}\right\rangle_{\beta, \infty}>0$. Then for large $\beta,\left\langle P_{\alpha}^{(1)} P_{\gamma}^{(2)}\right\rangle_{\beta, \infty}-\left\langle P_{\alpha}^{(1)}\right\rangle_{\beta, \infty}\left\langle P_{\gamma}^{(2)}\right\rangle_{\beta, \infty}$ cannot go to zero in the average, which would be required if $\langle\cdot\rangle_{\beta, \infty}$ were ergodic, so there are two or more phases, and $\left\langle P^{(2)}\right\rangle_{\beta, \infty} P^{(1)}-\left\langle P^{(1)}\right\rangle_{\beta, \infty} P^{(2)}$ will be a long range order parameter. Actually one can say more; namely if $\lim _{\beta \rightarrow \infty}\left\langle P_{\alpha}^{(i)}\right\rangle_{\beta, \infty}>0$ for $i=1, \ldots, k$ there will be, for $\beta$ large, at least $k$ phases; for, if \langle\rangle$_{A}$ were a convex combination of $k-1$ or fewer ergodic states, then

$$
a_{i j}=\lim _{\Lambda \rightarrow \infty}|\Lambda|^{-2} \sum_{\alpha, \beta \in \Lambda}\left\langle P_{\alpha}^{(i)} P_{\beta}^{(j)}\right\rangle_{\infty}
$$

would exist and would be a matrix of rank at most $k-1$ with $\sum_{j} a_{i j}=\left\langle P_{\alpha}^{(i)}\right\rangle$. Under the given supposition it has rank at least $k$ for $\beta$ large. See also $[9,5]$.

How does one show that $\left\langle P_{\alpha}^{(i)} P^{(j)}\right\rangle$ is small for $j \neq i$ ? Let $\Gamma$ be a contour in the elementary Peierls argument (see e.g. $[39,16])$ sense. Let $p_{i}(\Gamma)=$ probability that each spin immediately inside $\Gamma$ is in $K_{i}$ and each spin outside $\Gamma$ is not in $K_{i}$. Suppose that $p_{i}(\Gamma) \leqq e^{-C(\beta)|\Gamma|}$ with $C \rightarrow \infty$ as $\beta \rightarrow \infty$. Then, by the usual argument for cubes $\Lambda$ :

$$
\begin{aligned}
\left\langle P_{\alpha}^{(i)} P_{\gamma}^{(j)}\right\rangle & \leqq \sum_{\Gamma \text { around } \alpha} p_{i}(\Gamma)+\sum_{\Gamma \text { around } \beta} p_{i}(\Gamma)+\sum_{\substack{I \text { wrapped } \\
\text { around }|\Lambda|}} p_{i}(\Gamma) \\
& \leqq \sum_{|\Gamma|=2 v}^{\infty}(|\Gamma|+1)^{N} e^{d|\Gamma|} e^{-C(\beta)|\Gamma|}
\end{aligned}
$$

for suitable $d$ and $N$ independent of $\beta$ (but dependent on $v$ ). Thus to show that $\left\langle P_{\alpha}^{(i)} P_{\gamma}^{(j)}\right\rangle$ is small uniformly in $\alpha, \gamma$, and $\Lambda$ as $\beta \rightarrow \infty$, we only need to show that

$$
\left\langle\prod_{\alpha \text { inside } \Gamma} P_{\alpha}^{(i)} \prod_{\alpha \text { outside } \Gamma} P_{\alpha}^{\left(j_{\alpha}\right)}\right\rangle \leqq e^{-C_{0}(\beta)|\Gamma|}
$$


for any choice of the $j_{\alpha}$ 's (all distinct from $i$ ), for then

$$
(m-1)^{|\Gamma|} e^{-C_{0}(\beta)|\Gamma|} \equiv e^{-C(\beta)|\Gamma|} .
$$

Finally (4.5) is proven by using chessboard estimates, either directly in the form of Theorem 4.2 or an extended form of Theorem 4.2 which exploits a two site basic element. The net result is that the left side of (4.5) is dominated by the product of $|\Lambda|$ terms (or in the two site picture of $|\Lambda| / 2$ terms) most of which are 1 . But $0(|\Gamma|)$ of them are of the form $f \equiv\left\langle\prod_{\alpha \in \Lambda} P_{\alpha}^{\left(k_{\alpha}\right)}\right\rangle^{1 /|\Lambda|}$ where $\alpha \rightarrow k_{\alpha}$ is a function that has to be worked out in each case. Typically $f$ can be easily estimated to be small by energetic considerations. See $[14,9,5,19]$ and Paper II for explicit examples.

Of course, that leaves the questions of showing that

$\lim _{\beta \rightarrow \infty}\left\langle P_{\alpha}^{(i)}\right\rangle_{\Lambda=\infty}>0$ for several i's.

We discuss this in detail in Paper II, but note that this often follows from symmetry, or by applying the chessboard estimate to obtain an upper bound on $\left\langle\sum_{k \neq i} P_{\alpha}^{(k)}\right\rangle_{\Lambda=\infty}$ which is small, see also $[9,5,19]$.

Thus far, Peierls-type arguments have not been applicable in cases where a phase transition is accompanied by a spontaneously broken continuous symmetry. The only tool available is that invented in [10]: in the notation of Example 1 of $\S 3$, let $\sigma$ be a function on $K$, and let $\sigma_{\alpha}$ be the function $\sigma$ on the $\alpha$ th copy of $K$. For $\Lambda$ a cube, let $p$ be in $\Lambda^{*}$, the Fourier dual for $\Lambda\left(=1^{\text {st }}\right.$ Brillouin zone; $=$ dual group to $\Lambda$ viewed as a torus) and define

$$
\begin{gathered}
\hat{\sigma}_{p}=\frac{1}{\sqrt{|\Lambda|}} \sum_{\alpha \in \Lambda} e^{i p \alpha} \sigma_{\alpha} \\
g_{\Lambda}(p)=\left\langle\hat{\sigma}_{p} \hat{\sigma}_{-p}\right\rangle_{\beta, \Lambda} .
\end{gathered}
$$

Suppose that one can prove that for $p \neq 0$ :

$$
g_{\Lambda}(p) \leqq 1 / 2 \beta E_{p}
$$

for $E_{p}$ a function satisfying

$$
(2 \pi)^{-v} \int_{\substack{\left|p_{2}\right| \leqq \pi \\ i=1, \ldots, v}} E_{p}^{-1} d^{v} p \equiv C_{0}<\infty
$$

and that for $\beta \geqq \beta_{0}$

$$
\left\langle\sigma_{\alpha}^{2}\right\rangle \geqq D>0 \text {. }
$$

Then (following the version of the argument in [4]) for $\beta>\max \left(\beta_{0}, \beta_{1}\right)$ where $\beta_{1}=C_{0} / 2 D$, we will have (assuming some regularity on $E_{p}$ )

$$
\lim _{|\Lambda| \rightarrow \infty}\left[|\Lambda|^{-1} g_{\Lambda}(p=0)\right]>0
$$

since

$$
\begin{aligned}
|\Lambda|^{-1} g_{\Lambda}(p=0) & =|\Lambda|^{-1} \sum_{p \in \Lambda^{*}} g_{\Lambda}(p)-|\Lambda|^{-1} \sum_{p \neq 0} g_{\Lambda}(p) \\
& \geqq\left\langle\sigma_{\alpha}^{2}\right\rangle_{\Lambda}-|\Lambda|^{-1} \sum_{p \neq 0} 1 / 2 \beta E_{p},
\end{aligned}
$$


where the first sum is controlled by a Plancherel formula, and the second by (4.6). With minimal regularity assumptions on $E$,

$$
\lim _{\Lambda \rightarrow \infty}(4.10)=\left\langle\sigma_{\alpha}^{2}\right\rangle-(2 \beta)^{-1} C_{0}
$$

so (4.9) holds. By an argument of Griffiths (see e.g. [4]), (4.9) implies a first order phase transition with $\sigma_{\alpha}$ as order parameter.

In certain quantum cases (where $\sum_{\alpha \in A} \sigma_{\alpha}$ and $H$ do not commute) and, as we shall see below, for some other than simple, cubic lattices like the face centered cubic lattices, it is necessary or more convenient to rely not on (4.9) but rather on a direct infinite volume argument which is explained in detail in $[5,6,4]$.

We note that sometimes (4.8) follows by a symmetry argument (e.g. in the classical Heisenberg model) but that in general one can try to use a chessboard argument to show, e.g. that $\operatorname{Prob}\left(\sigma_{x}^{2} \leqq 2 D\right) \leqq 1 / 2$ for $\beta \geqq \beta_{0}$.

The only known way of proving (4.6) is via a "Gaussian domination" or

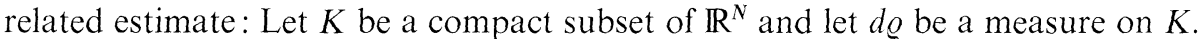
Let $\sigma^{(1)}, \ldots, \sigma^{(N)}$ be the coordinate functions on $K$. Suppose that $H$ has the form

$$
H=\frac{1}{2} \sum_{\alpha \neq \gamma} J_{\alpha \gamma}\left(\sigma_{\alpha}-\sigma_{\gamma}\right)^{2} \quad \text { (each pair counted once) }
$$

and define for $\left\{h_{\alpha \gamma}\right\}_{\alpha \neq \gamma}$ real,

$$
Z\left(h_{\alpha \gamma}\right)=\left\langle\exp \left[-\frac{1}{2} \beta \sum_{\alpha \neq \gamma} J_{\alpha \gamma}\left(\sigma_{\alpha}-\sigma_{\gamma}-h_{\alpha \gamma}\right)^{2}\right]\right\rangle_{0},
$$

where $\langle\cdot\rangle_{0}=\int \cdot \pi d \varrho\left(\sigma_{\alpha}\right)$ as usual. We claim that the two conditions: $J_{\alpha \gamma}=J_{\alpha-\gamma}$ $=J_{\gamma-\alpha}$ and

$$
Z\left(h_{x_{\gamma}}\right) \leqq Z(0) \quad \text { (Gaussian domination) }
$$

imply (4.6) with

$$
E_{p}=\frac{1}{2} \sum_{\alpha \in A}\left(1-e^{i p \cdot \alpha}\right) J_{\alpha 0}
$$

Before proving this, we note that one point of the Definition (3.5) is that it makes $E_{p}$ independent of $\Lambda$ for $p \in \Lambda^{*}$.

Since the argument to go from (4.11) to (4.6) is only a mild extension of that in [10], we only sketch the details. By translation invariance, $\left.\frac{d}{d \lambda} Z\left(\lambda h_{\alpha \gamma}\right)\right|_{\lambda=0}=0$ so that (4.11) implies that $\frac{d^{2}}{d \lambda^{2}} Z\left(\lambda h_{\alpha \gamma}\right)_{\lambda=0} \leqq 0$. This is equivalent to:

$$
\frac{1}{2} \beta^{2}\left\langle\left|\sum_{\alpha \neq \gamma} J_{\alpha \gamma}\left(\sigma_{\alpha}-\sigma_{\gamma}\right) \cdot h_{\alpha \gamma}\right|^{2}\right\rangle \leqq \frac{1}{2} \beta \sum_{\alpha \neq \gamma}\left|h_{\alpha \gamma}\right|^{2} J_{\alpha \gamma} .
$$


(4.13) only holds apriori for real $h_{x \gamma}$ but it extends to complex $h$. Now take $h_{x \gamma}=\left(e^{i p \cdot x}-e^{i p \cdot \gamma}\right)|\Lambda|^{-1 / 2}$ and find that (4.13) implies (4.8) with $E_{p}$ given by (4.12). We summarize:

Theorem 4.4. The Gaussian domination bound $Z\left(h_{\alpha \gamma}\right) \leqq Z(0)$ together with $J_{\alpha \gamma}=J_{\alpha-\gamma, 0}=J_{\gamma-\alpha, 0}$ implies the infrared bound $g_{\Lambda}(p) \leqq\left(2 \beta E_{p}\right)^{-1}$ with $E_{p}=\frac{1}{2} \sum_{\alpha \in \Lambda}\left(1-e^{i p \cdot \alpha}\right) J_{\alpha 0}$.

We next turn to a detailed investigation of (4.11).

Proposition 4.5. Suppose that $J_{x y} \geqq 0$. Then it suffices to check (4.11) for $h_{x y}$ of the form $h_{\alpha}-h_{\gamma}$.

Proof. Since $J_{x y} \geqq 0, Z \rightarrow 0$ as any $h_{x \gamma} \rightarrow \infty$ and thus $Z$ takes its maximum at some finite point. But $\partial Z / \partial h_{x \gamma}=0$ implies that

$$
J_{x \gamma}\left(h_{x \gamma}-\left\langle\sigma_{\alpha}-\sigma_{\gamma}\right\rangle\right)=0
$$

for the obvious expectation. Thus, letting $h_{\alpha} \equiv\left\langle\sigma_{\alpha}\right\rangle$, we see that $h_{\alpha \gamma}=h_{\alpha}-h_{\gamma}$ for those $\alpha \gamma$ with $J_{\alpha \gamma} \neq 0 . Z$ is independent of the other $h_{\alpha \gamma}$ so we can take $h_{\alpha \gamma}=h_{\alpha}-h_{\gamma}$ for such $(\alpha \gamma)$ without changing $Z$; i.e. $Z$ takes its maximum value at a point $h_{\alpha \gamma}=h_{\alpha}-h_{\gamma}$.

Remark. The proof of Theorem 4.4 only used (4.11) for the special case $h_{\alpha \gamma}=h_{\alpha}-h_{\gamma}$ so that Proposition 4.5 is, at this stage, primarily of academic interest. Indeed, there are $J_{\alpha \gamma}$ not all non-negative so that (4.11) holds for $h_{\alpha \gamma}$ of the form $h_{\alpha}-h_{\gamma}$, and thus (4.6) holds, even though for such $J$ 's, $Z \rightarrow \infty$ for a suitable choice of $h_{\alpha \gamma}$ not of the form $h_{x}-h_{\gamma}$.

It is an important and interesting open question to characterize the ferromagnetic interactions for which the spin 1/2 Ising model obeys Gaussian domination. We only have partial results on this question relying on reflection positivity. We begin with some examples which delimit the class and, in particular, demonstrate the falseness of the apriori attractive conjecture that Gaussian domination holds for all ferromagnets:

Example 1. Consider two spins, one $\sigma_{1}$, with values \pm 1 and the other, $\sigma_{2}$ with values \pm 2 , all values having equal apriori weight. Then $\left\langle e^{-J\left(\sigma_{2}-\sigma_{1}-h\right)^{2}}\right\rangle$ has its maximum near $h= \pm 1$ as $J \rightarrow \infty$. This shows that equality of the single spin distributions is essential for Gaussian domination in general (but see Examples 5 and 6).

Example 2. Let $\sigma_{1}= \pm 1, \sigma_{2}=+1$, then

$$
Z(h)=\frac{1}{2}\left[e^{-J h^{2}}+e^{-J(2-h)^{2}}\right]
$$

has its maximum near $h=1$ as $J \rightarrow 0$. The given distribution for $\sigma_{2}$ can be thought of as that of an Ising spin in an intense positive magnetic field. The failure of RP in this case shows that even equality of the magnetic fields at each point is also essential for Gaussian domination. 
Example 3. (Mean field model.) This is the most involved but also the most significant of the examples we present. $Z(h) \leqq Z(0)$ implies that $M_{\alpha \gamma}=-\left.\frac{\partial^{2} \ln Z}{\partial h_{\alpha} \partial h_{\beta}}\right|_{h_{\alpha}=0}$ is positive semi-definite. For a spin $1 / 2$ model with

$$
H=\frac{1}{2} \sum_{\gamma \neq \alpha} J_{\alpha_{i}}\left(\sigma_{\alpha}-\sigma_{\gamma}\right)^{2}
$$

a simple calculation shows that ( set $J_{\alpha \alpha}=0$ )

$$
M_{\alpha \gamma^{\prime}}=\delta_{\gamma \gamma}\left(\sum_{\delta} J_{\alpha \delta}\right)-J_{\alpha \gamma}-\sum_{\delta, \lambda} J_{\alpha \delta} J_{\gamma \lambda}\left\langle\left(\sigma_{\alpha}-\sigma_{\delta}\right)\left(\sigma_{\gamma}-\sigma_{j}\right)\right\rangle .
$$

Take $n+1$ spins, $\sigma_{0}, \ldots, \sigma_{n}$ with only $J_{0 i} \neq 0$, all equal to $\alpha_{n}$. Then

$$
M_{00}=n \alpha_{n}-\alpha_{n}^{2}\left[n^{2}+n+\left(n^{2}-n\right)\left\langle\sigma_{i} \sigma_{j}\right\rangle-2 n^{2}\left\langle\sigma_{0} \sigma_{i}\right\rangle\right] \text {. }
$$

Take $\alpha_{n}=1 / \sqrt{n}$, so that $H=-\sigma_{0}\left(\frac{1}{\sqrt{n}} \sum_{1}^{n} \sigma_{i}\right)+$ const and thus, as $n \rightarrow \infty$, a coupling of a Gaussian and a spin $1 / 2$ spin. Thus as $n \rightarrow \infty$

$$
\begin{aligned}
& \left\langle\sigma_{0} \sigma_{i}\right\rangle=\frac{1}{\sqrt{n}}\left\langle\sigma_{0} \frac{1}{\sqrt{n}} \sum_{i=1}^{n} \sigma_{i}\right\rangle \sim c / \sqrt{n} \\
& \left\langle\sigma_{i} \sigma_{j}\right\rangle=\frac{1}{n-1}\left[\left\langle\left(\frac{1}{\sqrt{n}} \sum_{1}^{n} \sigma_{i}\right)^{2}\right\rangle-1\right] \sim d / n
\end{aligned}
$$

for finite non-zero, $c$ and $d$. Thus

$$
M_{00}=-n-1+2 c \sqrt{n}+\sqrt{n}+0(1)
$$

is negative for $n$ large and therefore $M_{\alpha \gamma}$ is not positive definite for $n$ large.

Our next example, while a trivial extension of RP ideas illustrates that Gaussian domination can hold in some cases where RP fails :

Example 4. Let $\langle\cdot\rangle$ be an expectation for a string of $6 n$ spins with third neighbor ferromagnetic coupling. Then RP fails both for reflections about the midpoint of bonds and for reflections on sites. Since $Z(h)$ is a product of three nearest neighbor $2 n$-point $Z$ 's, Gaussian domination for that case yields it for the case at hand.

Our final three examples show that special features of the $J$ 's and/or the single spin distributions can allow one to prove Gaussian domination without RP and/or translation invariance. We hasten to add that phase transitions will not occur in Examples 5-7.

Example 5. Suppose that $H=\sum J_{\alpha \beta}\left(\sigma_{\gamma}-\sigma_{\beta}\right)^{2}$ with $J_{\alpha \beta}$ arbitrary positive numbers and that each single spin measures $d \varrho_{\alpha}\left(\sigma_{\gamma}\right)$ equals $F_{\gamma} d \sigma_{\gamma}$ with $F_{\gamma} \log$ concave and even, but not necessarily $\alpha$ independent. Since $e^{-H\left(\sigma_{\alpha}-h_{\alpha}\right)} \prod_{\alpha} F_{\alpha}$ is a log concave function of $\left\{\sigma_{\alpha}, h_{\alpha}\right\}, Z\left(h_{\alpha}\right)$ is log concave in $h_{\alpha}$ by a general theorem (see e.g. [1]). Since $\partial Z / \partial h_{\alpha}=0$, all $h_{\alpha}=0$, by symmetry, log concavity implies that $Z\left(h_{\alpha}\right) \leqq Z(0)$. 
Example 6. Suppose that $H=J_{\alpha \beta}\left(\sigma_{\alpha}-\sigma_{\beta}\right)^{2}$ with $J_{\alpha \beta}$ arbitrary positive numbers and that each single spin measure $d \varrho_{\alpha}\left(\sigma_{\alpha}\right)$ equals $F_{\alpha} d \sigma_{\alpha}$ with $F_{\alpha}$ positive definite and real (hence even), but not necessarily $\propto$ independent. Then

$$
\begin{aligned}
Z\left(h_{\alpha}\right) & =\int e^{-H\left(\sigma_{\alpha}-h_{\alpha}\right)} \prod_{\alpha}\left[\left(\int e^{i k_{\alpha} \sigma_{\alpha}} d \mu_{\alpha}\left(k_{\alpha}\right)\right) d \sigma_{\alpha}\right] \\
& =\int \prod_{\alpha} d \mu\left(k_{\alpha}\right) e^{i k_{\alpha} h_{\alpha}} \int \prod_{\alpha}\left(d \sigma_{\alpha} e^{i k_{\alpha} \sigma_{\alpha}}\right) e^{-H\left(\sigma_{\alpha}\right)}
\end{aligned}
$$

is positive definite in the $h$ 's since the Fourier transform of a Gaussian is a Gaussian. In particular, $Z\left(h_{x}\right)$ takes its maximum value at $h_{x}=0$ (in essence, the above calculation is proving that the convolution of positive definite functions is positive definite).

Example 7. Consider an array of $n$ spin $1 / 2$ Ising spins, $s_{1}, \ldots, s_{n}$ on a line with arbitrary positive, nearest neighbor couplings, $J_{12}, J_{23}, \ldots, J_{n 1}$. Let $T(J, h)$ be the two by two matrix

$$
\left(\begin{array}{cc}
e^{-1 / 2 J h^{2}} & e^{-1 / 2 J(2-h)^{2}} \\
e^{-1 / 2 J(2+h)^{2}} & e^{-1 / 2 J h^{2}}
\end{array}\right)
$$

i.e. if we label matrices as $\left(\begin{array}{ll}a_{++} & a_{+-} \\ a_{-+} & a_{--}\end{array}\right)$, then $T(J, h)_{\sigma_{1} \sigma_{2}}=\exp \left(-\frac{1}{2} J\left(\sigma_{1}-\sigma_{2}-h\right)^{2}\right)$. We want to note two critical facts about these matrices: first $T(J, 0)$ is positive definite and $T(J, 0)^{-1 / 2} T(J, h) T(J, 0)^{-1 / 2}$ is a contraction in the norm $\|(\alpha, \beta)\|$ $=\left(\alpha^{2}+\beta^{2}\right)^{1 / 2}$-this is proven in [10]. Secondly, the $T(J, 0)$ all commute, are positive definite and when diagonalized simultaneously their largest eigenvalues correspond to a common eigenvector-this follows by noting that $\frac{1}{\sqrt{2}}\left(\begin{array}{l}1 \\ 1\end{array}\right)$ and $\frac{1}{\sqrt{2}}\left(\begin{array}{r}1 \\ -1\end{array}\right)$ are the eigenvectors, and the eigenvalue for the first eigenvector is always largest. Since $Z\left(h_{1}, \ldots, h_{n}\right)=\operatorname{Tr}\left(T\left(J_{12}, h_{2}-h_{1}\right) \ldots T\left(J_{n 1}, h_{1}-h_{n}\right)\right)$ we can write

$$
Z\left(h_{1}, \ldots, h_{n}\right)=\operatorname{Tr}\left(A_{1} B_{1} \ldots A_{n} B_{n}\right),
$$

where $A_{i}=T\left(J_{i-1, i}, 0\right)^{1 / 2} T\left(J_{i, i+1}, 0\right)^{1 / 2}$ (where $\left.J_{01} \equiv J_{n 1}\right)$ and $B_{i}$ is a contraction, by the first fact noted above. Let $\mu_{1}(C), \ldots, \mu_{m}(C)$ be the singular values of an $m \times m$ matrix [eigenvalues of $\left(C^{*} C\right)^{1 / 2}$ ordered so that $\mu_{1} \geqq \mu_{2} \geqq--\geqq 0$ ]. An inequality of Horn [21] (see Corollary II.4.1 of [15]) asserts that

$$
\sum_{i=1}^{m} \mu_{i}\left(C_{1} \ldots C_{k}\right) \leqq \sum_{i=1}^{m} \mu_{i}\left(C_{1}\right) \ldots \mu_{i}\left(C_{k}\right) .
$$

Thus, we have that

$$
\begin{aligned}
Z\left(h_{1}, \ldots, h_{n}\right) & \leqq \sum_{i=1}^{2} \mu_{i}\left(A_{1} \ldots B_{n}\right) \\
& \leqq \sum_{i=1}^{2} \mu_{i}\left(A_{1}\right) \mu_{i}\left(A_{2}\right) \ldots \mu_{i}\left(A_{n}\right) \\
& =\sum_{i=1}^{2} \mu_{i}\left(A_{1} \ldots A_{n}\right)=Z(0, \ldots, 0),
\end{aligned}
$$


where we use the fact that $\mu_{j}\left(B_{i}\right) \leqq 1$, since $B_{t}$ is a contraction, in the second inequality, and use the second noted fact in the equality that follows.

To illustrate the close connection between chessboard estimates and Gaussian domination, we note:

Theorem 4.6. Let $\Lambda$ be a $2 n_{1} \times \ldots \times 2 n_{v}$ rectangle in $\mathbb{Z}^{v}$. Let $J_{x y}$ be a given on $\Lambda$ so that the chessboard estimate (Theorem 4.1) holds for $\langle\cdot\rangle=Z^{-1} \int \cdot e^{-H\left(\sigma_{\alpha}\right)} \prod_{\alpha \in A} d \varrho\left(\sigma_{z}\right)$
for all d@ in $\mathbb{R}^{N}$ and

$$
H=\frac{1}{2} \sum_{\alpha \neq \gamma} J_{\alpha \gamma}\left(\sigma_{\alpha}-\sigma_{\gamma}\right)^{2} .
$$

Then the Gaussian domination estimate $Z\left(h_{x}\right) \leqq Z(0)$ holds for arbitrary de and, in particular, $g_{\Lambda}(p) \leqq\left(2 \beta E_{p}\right)^{-1}$.

Proof. By a limiting argument, we can suppose that $d \varrho(\sigma)=F(\sigma) d^{N} \sigma$ with $F>0$ on all of $\mathbb{R}^{N}$. Then, if we define $G_{\alpha}(\sigma)=F\left(\sigma+h_{\alpha}\right) / F(\sigma)$ we have that

$$
\begin{aligned}
Z\left(h_{\alpha}\right) & \equiv \int e^{-H\left(\sigma_{\alpha}-h_{\alpha}\right)} \prod_{\alpha} d \varrho\left(\sigma_{\alpha}\right) \\
& =\int e^{-H\left(\sigma_{\alpha}\right)} \prod_{\alpha} d \varrho\left(\sigma_{\alpha}+h_{\alpha}\right) \\
& =Z(0)\left\langle\prod_{\alpha} G_{\gamma}\left(\sigma_{\alpha}\right)\right\rangle \\
& \leqq Z(0) \prod_{\alpha}\left\langle\prod_{\beta} G_{\alpha}\left(\sigma_{\beta}\right)\right\rangle^{1 /|\Lambda|} \\
& =\prod_{\alpha}\left[\int^{-H\left(\sigma_{\gamma}\right)} \prod_{\gamma} d \varrho\left(\sigma_{\gamma}+h_{\alpha}\right)\right]^{1 /|A|}=Z(0),
\end{aligned}
$$

where the inequality is a chessboard estimate and the last equality comes from $H\left(\sigma_{\alpha}-h\right)=H\left(\sigma_{\alpha}\right)$ for constant $h$.

Remark. Using the Dobrushin-Lanford-Ruelle equations one can prove Theorem 4.6 directly in infinite volume for RP Gibbs states.

The above argument has a defect: it does not obviously extend to the quantum case.

Fortunately, one can use a version of the original argument given in [10], based on Theorem 2.3: Namely, in the case of $2 n$ spins, Theorem 2.3 says that

$$
\begin{aligned}
\left|Z\left(h_{-n+1}, \ldots, h_{n}\right)\right|^{2} \leqq & Z\left(h_{-n+1}, \ldots, h_{0}, h_{0}, h_{-1}, \ldots, h_{-n+1}\right) \\
& \cdot Z\left(h_{n}, h_{n-1}, \ldots, h_{1}, h_{1}, \ldots, h_{n}\right)
\end{aligned}
$$

so that translation invariance and the argument in Theorem 4.1 show that $\max \left|Z\left(h_{i}\right)\right|$ occurs when all $h$ 's are equal. Since $Z(h, \ldots, h)=Z(0)$, the maximum is $Z(0)$. As of now, this is the most widely applicable proof of Gaussian domination we know of.

We remind the reader that in the quantum case there is one additional complication in that Gaussian domination does not lead to a bound on $\left\langle\hat{\sigma}_{p} \hat{\sigma}_{-p}\right\rangle$ but rather on a "Duhamel two point function", $\left(\hat{\sigma}_{p}, \hat{\sigma}_{-p}\right)$. This problem and its resolution are discussed in [4], for the case of nearest neighbor interactions. The present generalization is straight forward. 
The argument based on Theorem 2.3 has an additional advantage, even in the classical case. Suppose that $\tilde{H}=\frac{1}{2} \sum_{\alpha \neq \gamma} J_{\alpha \gamma}\left(\sigma_{\gamma}-\sigma_{\gamma}\right)^{2}+H^{\prime}$ where $\langle\cdot\rangle_{H^{\prime}}$ is RP and $J$ obeys (3.3). If $Z(h)=\left\langle\exp -\left(\frac{1}{2} \sum J_{\gamma_{\gamma}}\left(\sigma_{\alpha}-\sigma_{\gamma}-h_{\alpha}+h_{\gamma}\right)^{2}+H^{\prime}\right)\right\rangle_{0}$ then, as above, Theorem 2.3 implies that $Z\left(h_{x}\right) \leqq Z(0)$, and infrared bounds follow. We summarize with

Theorem 4.7. Let $H$ have the form of Theorem 4.6 with $J$ RP. Let $\tilde{H}=H+H^{\prime}$ with $H^{\prime} R N$. Let $Z\left(h_{\alpha}\right)=\left\langle\exp \left(H\left(\sigma_{\alpha}-h_{\alpha}\right)+H^{\prime}\right)\right\rangle_{0}$. Then $Z\left(h_{\alpha}\right) \leqq Z(0)$ and $g_{\Lambda}(p) \leqq\left(2 \beta E_{p}\right)^{-1}$ with $E_{p}$ depending on $J_{x,}$, as in Theorem 4.4.

Finally, we want to mention a problem (and its resolution) that occurs for certain special models like the ones on face centered cubic lattices. The infinite volume lattice is reflection invariant about any plane which is the perpendicular bisector of a bond, but any finite volume cutoff will destroy many of these symmetries. The resolution is the following: Let $\langle\cdot\rangle$ denote an infinite volume expectation and, given, $\left\{h_{x}\right\}_{\gamma \in \mathbb{Z}^{v}}$ with only finitely many non-zero $h_{\gamma}^{\prime}$ 's, let

$$
g\left(h_{\gamma}\right) \equiv\left\langle\exp \left(\frac{1}{2} \sum_{\alpha \neq \gamma} J_{\gamma_{\gamma}}\left[\left(\sigma_{\gamma}-\sigma_{\gamma}\right)^{2}-\left(\sigma_{\gamma}-\sigma_{\gamma}-h_{\alpha}+h_{\gamma}\right)^{2}\right]\right)\right\rangle .
$$

If we can show that $\left|g\left(h_{\alpha}\right)\right| \leqq 1$ for all $h_{\alpha}$, then by following the arguments in [10] one will get infinite volume infrared bounds and therefore long range order. To prove that $\left|g\left(h_{x}\right)\right| \leqq 1$, one need only show that $\langle\cdot\rangle$ has a kind of RP about each "bond" plane, i.e. that

$$
\left|g\left(h_{x}\right)\right|^{2} \leqq g\left(h_{x}^{\prime}\right) g_{x}\left(h_{x}^{\prime \prime}\right)
$$

where $h_{x}^{\prime}\left(\right.$ resp $\left.h_{x}^{\prime \prime}\right)$ is obtained by taking $h_{x}$ on the left (resp. right) side of the plane and reflecting in the plane. Given (4.14) it is not hard to reduce the proof of $\left|g\left(h_{\alpha}\right)\right| \leqq 1$ to showing that $\left|g\left(h_{\alpha}\right)\right|^{1|1 \Lambda|} \rightarrow 1$ for a set of $h_{\alpha}^{\prime}$ 's constant at $h_{0}$ on a nice set 1. But it is easy to see that $\left|g\left(h_{\gamma}\right)\right| \leqq e^{c|i A|}$ for such $h$ 's. (Instead one can use Theorem 4.6 in infinite volume; see e.g. [6]).

We can see two ways of proving (4.14). In cases where correlation inequalities are available, one can prove (4.14) for a given plane by taking a suitable sequence of "+ boundary condition states" where the given plane cuts $A$ exactly in half. Since the limit is independent of the sequence, (4.14) holds for the + boundary condition state. When correlation inequalities are not available, one can at least prove there are multiple phases; for, if not, then all periodic states converge to a unique state which would then obey (4.14). If $\left\langle\sigma_{\alpha}^{2}\right\rangle_{\beta, \infty}$ has a lower bound that is uniform in $\beta$ one would obtain long range order: a contradiction!

\section{Long Range Models}

In [3], Dyson showed that a spin $1 / 2$ Ising model with $J(n)=(1+|n|)^{-\alpha}$ has a phase transition if $1<\alpha<2(\alpha>1$ is needed for sensible thermodynamics), and did not if $2<\alpha$. His method works for any classical model with correlation inequalities such as the plane rotor model [13]. Using similar ideas, Kunz and Pfister [26] treated 
the two dimensional plane rotor model with $J(n)=(1+|n|)^{-\gamma}$, proving a phase transition if $2<\alpha<4$.

In this section, we illustrate the general methods of this paper by recovering these results (many more examples are presented in $[7,8]$ ) and extending them in several directions: a) cases where correlation inequalities are unknown such as the classical Heisenberg model can be accomodated; b) logarithmic improvements in Dyson's conditions are given; c) certain quantum models are accomodated.

We give details in the one dimensional classical case and then treat two dimensions and quantum models in a few remarks. When correlation inequalities of Griffiths type are available, improvements of our results of the following sort are possible: If a phase transition is known for an RP $J_{0}$ which is also positive, it holds for any larger $J$ even if the larger $J$ is not $R P$. We suppose in all cases that $\sum|J(n)|<x$.

We begin our analysis with:

Theorem 5.1. Let $K$ be a compact subset of $\mathbb{R}^{N}$ and let do be a measure different from $\delta(\sigma)$, invariant under $\sigma \rightarrow-\sigma$. Let $-\beta H=\beta \sum_{i>1} J(i-j) \sigma_{i} \cdot \sigma_{j}$ and let $E_{p}=\sum_{n=1}^{\infty} J(n)(1-\cos p n)$. If $0 \leqq J(n)$ and $J$ is $R P$, and if $g \equiv \int d p / E_{p}<\infty$, then there is a first order phase transition with $\sigma$ as order parameter, at some sufficiently large, finite $\beta$.

Theorem 5.2. Let $J(i-j)$ be RP. Then the classical isotropic Heisenberg model has a first order phase transition for $\beta$ large if and only if $g \equiv \int d p / E_{p}<\infty$.

Proofs. The absence of a first order phase transition (asserted in Theorem 5.2) if $g=\propto$ follows from a slight extension of an argument of Mermin [29], so we concentrate on the existence question. Since $g<x$, this follows, according to the strategy of $\S 4$, if we show that $\left\langle\hat{\sigma}_{p} \hat{\sigma}_{-p}\right\rangle_{\text {periodic }} \leqq 1 / 2 \beta E_{p}$ and $\lim _{\beta \rightarrow \infty}\left\langle|\sigma|^{2}\right\rangle_{\beta, x}>0 . J$ being RP implies that $\langle\cdot\rangle_{\text {periodic }}$ is RP by Theorems 2.1 and 3.4. The method of $\$ 4$ then yields the infrared bounds. In the case of Theorem 5.2, $\left\langle|\sigma|^{2}\right\rangle_{x_{\infty}}=1$ while in the case of Theorem 5.1, choose $r_{0}>0$ so that $\int_{|\sigma|>r_{0}} d \varrho>0$ and use a chessboard estimate to see that $\left\langle\left(|\sigma| \leqq r_{0}\right)\right\rangle_{\beta, \alpha} \rightarrow 0$, as $\beta \rightarrow \infty$. The right side of this chessboard estimate is controlled by noting that RP implies that the ground state with the restriction $\left|\sigma_{x}\right| \leqq r_{0}$ has all spins equal, and then by noting that the energy when all $\sigma_{x}=\boldsymbol{r}$ is strictly monotone increasing in $|\boldsymbol{r}|$, since $J(n) \geqq 0$.

These theorems reduce the study of the long range one dimensional case to the study of two questions: 1) When is $J$ RP? 2) When is $\int E_{p}^{-1} d p<\infty$. In studying the first question the following is useful:

Definition. A distribution $F$ on $\mathbb{R}^{\prime} \backslash\{0\}$ is called $O S$ positive (for OsterwalderSchrader [30]) if and only if $F$ is continuous and

$$
\int F(x-y) g(x) \tilde{g}(y) d x d y \geqq 0
$$

for all $g \in C_{0}^{\infty}\left(x_{1}>0\right)$ where $\tilde{g}\left(y_{1}, \ldots, y_{v}\right)=\overline{g\left(-y_{1}, y_{2}, \ldots, y_{v}\right)}$. 
Theorem 5.3. a) If $F$ is an $O S$ positive distribution on $R^{v}$ then $J$, defined on $\left\{\left(n_{1}, \ldots, n_{v}\right) \mid n_{1}>0\right\}$ by

$$
J(n)=F\left(n_{1}, \ldots, n_{v}\right) ; \quad n_{1}>0,
$$

is $R P$.

b) If $J_{1}$ and $J_{2}$ are $R P$ on $\left\{n_{1}>0\right\} \subset \mathbb{Z}^{v}$, then so is $J_{1} J_{2}$.

c) If $J(n)=\int_{0}^{\infty} e^{-n y} d \varrho(y),(n \geqq 1)$, then $J$ is $R P$ on $\mathbb{Z}^{1}$.

Proof. a) In (5.1), let $g$ approach a sum of delta functions. This shows at once that $J$ is RP.

b) Follows from the fact (Schur's theorem) that if $a_{i j}$ and $b_{i j}$ are positive definite matrices, so is $c_{i j}$ with $c_{i j}=a_{i j} b_{i j}$

c) A restatement of Proposition 3.2; it also follows from a) and well-known structure theorems for OS positive distributions.

Proposition 5.4. The following functions on $\mathbb{Z}$ are $R P$ in the region $n \geqq 1$ :
a) $J(n)=n^{-\alpha}$,
b) $J(n)=(1+n)^{-\alpha}$

for all $x>0$.

Proof.
a) $\int_{0}^{\infty} e^{-n y} y^{\alpha-1} d y=\Gamma(\alpha) n^{-\alpha}$
[use Theorem 5.3c];
b) $\int_{0}^{\infty} e^{-n v} e^{-v} y^{\gamma-1} d y=\Gamma(\alpha)(n+1)^{-\gamma}$
[use Theorem 5.3c].

As for the second question, we note:

Theorem 5.5. Let $E_{p}=\sum_{n=1}^{\infty} J(n)(1-\cos p n)$ with $J(n) \geqq 0$. Then

a) If $\sum_{n=1}^{\infty} n^{-3} J(n)^{-1}<\infty$, then $\int d p E_{p}^{-1}<\infty$.

b) If $\limsup _{N \rightarrow \infty}(\log N)^{-1}\left[\sum_{1}^{N} n J(n)\right]<\infty$, then $\int d p E_{p}^{-1}=\infty$.

Remarks. 1. The condition in a) is slightly weaker than the one that Dyson [3] needs for a phase transition. The condition in b) is slightly weaker than the one that Dyson [3] needs to prove that there is no phase transition in the Ising model; b) will only imply the absence of continuous symmetry breaking. This is as it must be if the $n^{-2}$ Ising model has a phase transition (as is believed), since $J(n)=n^{-2}$ obeys the conditions of $b$ ).

2. b) includes the case $J(n)=n^{-2}$. This case can be done by explicit calculation of $E_{p}$ (contained in the tables, e.g. (516) of [24]) or by noting that $E_{p}=f(0)-f(p)$ with $f(p)=\sum_{1}^{\infty} n^{-2} \cos p n$ obeying $f^{\prime \prime}(p)=\pi \delta(p)-\frac{1}{2}$ with periodic boundary conditions at $\pm \pi$. One sees that $E(p) \sim|p|$ in that case.

3. If $J(n) \sim n^{-\alpha}$ at infinity, we are in case (a) if $\alpha<2$ and in case b) if $\alpha \geqq 2$. Actually with regard to a) one cannot improve even logs, since for $J(n)$ 
$\sim n^{-2}(\log n) \ldots\left(\log _{m} n\right)^{1+\varepsilon}$, then $E_{p} \sim|p|(\log p) \ldots\left(\log _{m} p\right)^{1+\varepsilon}$. For b), improvements are presumably possible: with little change $(\log N)^{-1}$ can be replaced by $\left[(\log N)\left(\log _{2}\right)(N) \ldots \log _{m}(N)\right]^{-1}$ which allows only $n^{-2}\left(\log _{2} n\right) \ldots\left(\log _{m} n\right)^{1+\varepsilon}$.

4. If $J(n)=\int_{-1}^{1} \lambda^{|n|-1} d \varrho$, then $E_{p} \equiv \sum_{n=1}^{\infty} J(n)(1-\cos p n)$ increases when $d \varrho$ increases.

This remark allows one to obtain results for $J$ 's which are RP but not positive from those in this theorem.

Proof. a) We need a lower bound on $E_{p}=\sum_{1}^{\infty} J(n)(1-\cos n p)$. For $|x| \leqq \pi,(1-\cos x)$ $\geqq \frac{2}{\pi^{2}} x^{2}$ so that

$$
E_{p} \geqq \sum_{1}^{[\pi /|p|]} \frac{2}{\pi^{2}} p^{2} n^{2} J(n)
$$

where $[x]=$ greatest integer less than $x$. Thus we need only show that

$$
\alpha \equiv \int_{0}^{\pi} d p\left[\sum_{1}^{[\pi / p \mid]} p^{2} n^{2} J(n)\right]^{-1}<\infty .
$$

By the Schwarz inequality

$$
\begin{aligned}
{[\pi / p]^{2} } & =\left(\sum_{1}^{[\pi / p]} 1\right)^{2} \\
& \leqq\left[\sum_{1}^{[\pi / p]} n^{2} J(n)\right]\left[\sum_{1}^{[\pi / p]}\left(n^{2} J(n)\right)^{-1}\right]
\end{aligned}
$$

so that

$$
\begin{aligned}
\alpha & \leqq \int_{0}^{\pi} d p p^{-2}[\pi / p]^{-2} \sum_{1}^{[\pi / p]}\left(n^{2} J(n)\right)^{-1} \\
& \leqq \frac{4}{\pi^{2}} \int_{0}^{\pi} d p \sum_{1}^{[\pi / p]}\left(n^{2} J(n)\right)^{-1}
\end{aligned}
$$

since $n^{2}[n]^{-2} \leqq([n]+1)^{2}[n]^{-2} \leqq 4$ for $n \geqq 1$ and $\pi / p \geqq 1$ for $0 \leqq p \leqq \pi$. Finally we note that

$$
\begin{aligned}
\int_{0}^{\pi} d p \sum_{1}^{[\pi / p]}\left(n^{2} J(n)\right)^{-1} & =\sum_{n=1}^{\infty}\left(n^{2} J(n)\right)^{-1} \int_{0}^{\pi / n} d p \\
& =\pi \sum_{1}^{\infty} n^{-3} J^{-1} .
\end{aligned}
$$

b) We need an upper bound on $E_{p}$. Since $(1-\cos x) \leqq|x|$ we have that

$$
E_{p} \leqq|p| \sum_{1}^{N} n J(n)+2 \sum_{N}^{\infty} J(n)
$$


for any $N$. To estimate the second term, let $K(j)=\sum_{1}^{j} n J(n)$ so that

$$
\begin{aligned}
\sum_{N}^{M} J(n) & =\sum_{N}^{M} \frac{1}{n}[K(n)-K(n-1)] \\
& =\sum_{n=N}^{M}\left[\frac{1}{n} K(n)-\frac{1}{n-1} K(n-1)\right]+\frac{1}{n(n-1)} K(n-1) \\
& =\frac{1}{M} K(M)-\frac{1}{N-1} K(N-1)+\sum_{N}^{M} \frac{1}{n(n-1)} K(n-1) .
\end{aligned}
$$

Thus, if $\frac{1}{M} K(M) \rightarrow 0$ as $M \rightarrow \infty$, we have that

$$
\sum_{N}^{\infty} J(n) \leqq \sum_{N=1}^{\infty} \frac{1}{n(n+1)} K(n) .
$$

If $K(n) \leqq C \log n$, we see that

$$
E_{p} \leqq C|p| \log N+\tilde{C} N^{-1} \log N .
$$

Choosing $N=\left[|p|^{-1}\right]$, we see that $\left.E_{p} \leqq C|p| C \log \left(|p|^{-1}\right)\right)$, so that $\int E_{p}^{-1} d p=\propto$.

By combining the previous results of this section we conclude that

Theorem 5.6. If $d \varrho \neq \delta(p)$ is a measure on $\mathbb{R}^{N}$ symmetric under $\sigma \rightarrow-\sigma$ and $J(n)=n^{-x}$, then there is a first order phase transition for the one dimensional spin model when $1<\alpha<2$.

Remark. If $N=1$ (or if $d \varrho$ is anisotropic in a suitable sense) but do is not even, there will be a phase transition in suitable external magnetic field when $1<\alpha<2$; see [10] or [7].

We describe the extensions in a series of remarks:

A) In two dimensions, the functions $p^{\alpha-1}$ have OS positive Fourier transforms for $x>-1$. This follows from

$$
\int_{0}^{x} \frac{d m}{p^{2}+m^{2}} m^{\alpha}=p^{x-1} \int_{0}^{x} \frac{x^{x} d x}{x^{2}+1}
$$

and the fact that $\left(p^{2}+m^{2}\right)^{-1}$ has an OS positive Fourier transform (free Euclidean field $[30,42])$. Since $x^{-\beta}(0<\beta<2)$ has a Fourier transform $c_{\beta} p^{2-\beta}$, we see that $|n|^{-\beta}$ is RP for $0<\beta<2$ by Theorem 5.3a). Then by Theorem 5.3b), we conclude that $|n|^{-\beta}$ is RP for all $\beta>0$. Calculations similar to those above show that in 2 dimensions, $\int d p / E_{p}<\propto$ if $\sum_{n \neq 0} n^{-6} J(n)^{-1}<\infty$; and for $J(n)=n^{-4}$, an explicit calculation involving periodic Green's functions for $-\Delta$ [and the fact that $\Delta\left(r^{-2}\right) \sim r^{-4}$ at $\left.x\right]$ shows that $E_{p} \sim p^{2} \log p+0\left(p^{2}\right)$ at $p=0$, so $\int d p / E_{p}=x$ in that case. We thus obtain:

Theorem 5.7. If $d Q \neq \delta(p)$ is a measure on $\mathbb{R}^{N}$ symmetric under $\sigma \rightarrow-\sigma$, and $J(n)=n^{-\alpha}$, then there is a first order phase transition for $2<x<4$, in the twodimensional spin model. 
This result is of interest only in isotropic cases.

B) It is easy to prove first order phase transitions in suitable quantum systems which are simultaneously real by using the method of [4]. In order for that method to be applicable one must check an algebraic condition; in particular some double commutator should not be large. There are two cases where this condition is easy to verify: in anisotropic models, such as $\sigma_{x} \sigma_{x}+\varepsilon \sigma_{y} \sigma_{v}$ with $\varepsilon<1$, the double commutator is always small at low temperatures, and in a classical limit, like $S \rightarrow \infty$ in Heisenberg models, the double commutator is small, for $S$ sufficiently large, [4]. We conclude:

Theorem 5.8. Fix $J(n)=n^{-x}$ for $1<\alpha<2$. Then the isotropic antiferromaynet with $-H=\sum_{n \neq m}(-1)^{n-m} J(|n-m|) \boldsymbol{S}_{n} \cdot \boldsymbol{S}_{m}$ for quantum spins $\boldsymbol{S}_{n}$ of spin $S$ has a first order phase transition if $S$ is sufficiently large (at some $\beta$ sufficiently large). Moreover, for any $\varepsilon$ with $0<\varepsilon<1$, the spin $1 / 2$ model with $-H=\sum_{n \neq m} J(|n-m|)\left(S_{n}^{x} S_{m}^{x}+\varepsilon S_{n}^{y} S_{m}^{y}\right)$ has a first order phase transition at some $\beta$ sufficiently large.

Acknowledgements. It is a pleasure to thank F. Dyson, O. Heilmann, L. Rosen, E. Seiler, J. Slawny, and T. Spencer for valuable discussions.

\section{References}

1. Brascamp,H., Lieb, E.H.: Some inequalities for Gaussıan measures and the long range order of the one dimensional plasma. In: Functional integration and its applications (ed. A. M. Arthurs). pp. 1-14. Oxford: Clarendon Press 1975

2. Donoghue,W.F., Jr.: Monotone matrix functions and analytic continuation. Berlin-HeidelbergNew York: Springer 1974

3. Dyson, F.: Existence of a phase transition in a one-dimensional ising ferromagnet. Commun. math. Phys. 12, 91 (1969). Non-existence of spontaneous magnetization in a one-dimensional ising ferromagnet. Commun. math. Phys. 12, 212 (1969)

4. Dyson, F., Lieb, E.H., Simon, B.: Phase transitions in quantum spin systems with isotropic and nonisotropic interactions. J. Stat. Phys. 18, 335-383 (1978). See also: Phase transitions in the quantum Heisenberg model. Phys. Rev. Letters 37, 120-123 (1976)

5. Fröhlich,J.: Phase transitions, goldstone bosons and topological superselection rules. Acta Phys. Austriaca Suppl. XV, 133-269 (1976)

6. Frohlich,J.: The pure phases (harmonic functions) of generalized processes. Or: mathematical physics of phase transitions and symmetry breaking. Invited talk at Jan. 1977 A.M.S., St. Louis meeting. Bull. Am. Math. Soc. (in press)

7. Fröhlich,J., Israel, R., Lieb, E.H., Simon, B.: Phase transitions and reflection positivity. II. Short range lattice models. J. Stat. Phys. (to be submitted)

8. Fröhlich,J., Israel, R., Lieb,E.H., Simon, B.: Phase transitions and reflection positivity. III. Continuous models. Commun. math. Phys. (to be submitted)

9. Fröhlich,J, Lıeb, E. H.: Phase transitions in anısotropıc lattıce spin systems. Commun. math. Phys 60, $233-267(1978)$

10. Fröhlich,J., Simon,B., Spencer, T.: Infrared bounds, phase transitions, and continuous symmetry breaking. Commun. math. Phys. 50, 79 (1976)

11. Gallavotti,G., Miracle-Sole, S. : Equilibrium states of the Ising model in the two-phase region. Phys. Rev. 5, 2555-2559 (1972)

12. Gelfand,I.M., Vilinkin, N. Ya.: Generalızed functions, Vol. 4. New York: Academic Press 1964

13. Ginibre,J. : General formulation of Griffiths' inequality. Commun. math. Phys. 16, 310-328 (1970)

14. Glimm, J., Jaffe, A., Spencer, T. : Phase transitions for $\phi_{2}^{4}$ quantum fields. Commun. math. Phys. 45 , $203(1975)$

15. Gohberg,I.C., Krein,M.G.: Introduction to the theory of linear non-selfadjoint operators. Providence, RI: American Mathematical Society 1969 
16. Griffiths, R. : Phase transitıons. In: Statıstical mechanics and quantum field theory, Les Houches, 1970. pp. 241-280. New York: Gordon and Breach 1971

17. Hegerfeldt, G.C.: Correlation inequalitıes for Ising ferromagnets with symmetries. Commun. math. Phys. 57, 259-266 (1977)

18. Hegerfeldt,G.C., Nappi,C.: Mixing properties in lattice systems. Commun. math. Phys. 53, $1-7$ (1977)

19. Heilmann,O.J., Lieb, E.H.: Lattice models for liquid crystals (1n preparation)

20. Holsztynski,W., Slawny, J. : Peierls condition and number of ground states. Commun. math. Phys. 61, 177--190(1978)

21. Horn, A. : On the singular values of a product of completely continuous operators. Proc. Nat. Acad. Sc1. USA 36, $374-375$ (1950)

22. Israel, R.: Convexity and the theory of lattice gases. Princeton, NJ: Princeton University Press 1978

23. Israel, R. : Phase transitions in one-dimensional lattice systems. Proc. 1977 IUPAP Meeting, Haifa

24. Jolley, C.B.W.: Summation of series. New York: Dover 1961

25. Klein, A. : A characterization of Osterwalder-Schrader path spaces by the associated semigroup. Bull. Am. Math. Soc. 82, 762 - 764 (1976)

26. Kunz,H., Pfister, C. E. : First order phase transition in the plane rotor ferromagnetic model in two dimensions. Commun. math. Phys. 46, 245 (1976)

27. Lieb,E.H.: New proofs of long range order. Proceedings of the International Conference on the Mathematical Problems in Theoretical Physıcs, Rome, 1977. Lecture notes in physics. BerlinHeidelberg-New York: Springer (in press)

28. Elliott, R.J.: Phenomenological discussion of magnetic ordering in the rare-earth metals. Phys. Rev. 124, 346-353 (1961)

29. Mermin, N.D.: Absence of ordering in certain classical systems. J. Math. Phys. 8, 1061 - 1064 (1967)

30. Osterwalder, K., Schrader. R. Axioms for Euclidean green's functions. Commun math. Phys. 31, $83(1973)$

31. Osterwalder. K., Seiler, E.: Gauge field theories on the lattice. Ann. Phys. 110, 440-471 (1978)

32. Kim,D., Thompson, C.J.: A lattice model with an infinite number of phase transitıons. J. Phys. A : Math. Gen. 9, 2097-2103 (1976)

33. Pirogov, S. A., Sinai, Ya.G. : Phase transitions of the first kind for small perturbations of the Ising model. Funct. Anal. Pril. 8, 25-30 (1974). [Engl. translation: Funct. Anal. Appl. 8, 21-25 (1974)]

34. Pirogov, S. A., Sinai, Ya.G. : Phase diagrams of classical lattice systems. Teor. Mat. Fiz. 25, 358 369 (1975). [Engl. translation: Theor. Math. Phys. 25, 1185-1192 (1975)]

35. Pirogov, S. A., Sina1, Ya.G.: Phase diagrams of classical lattice systems. Continuatıon. Theor. Mat. F1z. 26, 61-76 (1976). [Engl. translation: Theor. Math. Phys. 26, 39-49 (1971)]

36. Redner,S., Stanley,H.E : The R-S model for magnetic systems with competing interactions: series expansions and some rigorous results. J. Phys. C. Solid State Phys. 10, $4765 \sim 4784$ (1977)

37. Reed, M., Simon, B.: Methods of modern mathematical physics. Vol. Il: Fourier analysis, selfadjointness. New York: Academic Press 1975

38. Reed,M., Simon, B.: Methods of modern mathematical physics. Vol. IV: Analysis of operators. New York: Academic Press 1978

39. Ruelle,D.: Statistical mechanics. New York: Benjamin 1969

40. Schoenberg, I.J.: Metric spaces and positive definite functions. Trans. Am. Math. Soc. 44, $522-536$ (1938)

41. Schrader, R. : New Correlation inequalities for the Ising model and $P(\phi)$ theories. Phys. Rev. B 15, $2798-2803(1977)$

42. Simon, B. : The $P(\phi)_{2}$ Euclidean (quantum) field theory. Princeton, NJ : Princeton University Press 1974

43. Simon, B.: New rigorous existence theorems for phase transitions in model systems. Proc. 1977 IUPAP Meeting, Haifa

Communicated by J. Glimm

Received April 24, 1978 